\title{
Random graphs with bounded maximum degree: asymptotic structure and a logical limit law
}

\author{
Vera Koponen $\|^{\dagger}$ \\ Department of Mathematics, Uppsala University, Sweden \\ received $12^{\text {th }}$ March 2012, accepted $18^{\text {th }}$ October 2012.
}

For any fixed integer $R \geq 2$ we characterise the typical structure of undirected graphs with vertices $1, \ldots, n$ and maximum degree $R$, as $n$ tends to infinity. The information is used to prove that such graphs satisfy a labelled limit law for first-order logic. If $R \geq 5$ then also an unlabelled limit law holds.

Keywords: random graph, bounded degree, finite model theory, limit law

\section{Introduction}

This article is concerned with finite graphs with $n$ vertices labelled $1, \ldots, n$ such that every vertex has degree at most $R$, where $R$ will be a fixed non-negative integer. By graph we always mean finite undirected graph. The asymptotic structure of such graphs have been studied before, in numerous articles, in the case when a degree sequence is associated and one considers (only) graphs with this degree sequence. Two variants of degree sequences have been considered. In one variant a sequence $\bar{d}=\left(d_{1}, \ldots, d_{n}\right)$ of non-negative integers is given and one studies the graphs with vertices $1, \ldots, n$ such that, for every $i=1, \ldots, n$, the vertex $i$ has degree $d_{i}$. In the other variant we are given a sequence $\bar{d}=\left(d_{0}, d_{1}, \ldots, d_{R}\right)$ and study the graphs with vertices $1, \ldots, n$ such that exactly $d_{i}$ vertices have degree $i$. Early work on graphs with prescribed degree sequence include [2, 3, 17, 18, 13]. In particular one can consider degree sequences $\left(d_{1}, \ldots, d_{n}\right)$ in which all entries are the same, say $r$, in which case one gets $r$-regular graphs. This special case has also been studied extensively, for instance as a special case in the mentioned articles. See [19] for a survey about random regular graphs. Suppose that for all sufficiently large integers $n, \mathbf{G}_{n}$ is a set of graphs with vertices $1, \ldots, n$. We say that $\mathbf{G}_{n}$ satisfies a labelled logical limit law if for every property which can be expressed with a first-order sentence in the language of graphs, the proportion of graphs in $\mathbf{G}_{n}$ which have this property converges as $n \rightarrow \infty$. If convergence holds in the case when we count graphs only up to non-isomorphism, then we say that $\mathbf{G}_{n}$ satisfies an unlabelled limit law. If the number to which the mentioned proportion converges is always 0 or 1 , then one says that a zero-one law

\footnotetext{
$\dagger$ Email: Vera.Koponen@math.uu.se 
holds. Lynch [12] has proved that a labelled logical limit law holds if we consider an infinite sequence of degree sequences $\left(\bar{d}_{n}\right)_{n=1}^{\infty}$ (of the first kind mentioned above) which satisfies certain properties and for each $n$ the graphs with vertices $1, \ldots, n$ that have the degree sequence $\bar{d}_{n}=\left(d_{n, 1}, \ldots, d_{n, n}\right)$. In particular, the main result in [12] implies that $r$-regular graphs satisfy a labelled limit law, but not a zero-one law. One of the assumed properties of the sequence of degree sequences in [12] fails in the instances that turn out to be of interest in this article, so we cannot use [12]. Haber and Krivelevich [7] have proved zero-one laws, and the absence of a limit law, for $r(n)$-regular graphs when $r(n)$ is a function of the form $\alpha n$ or $n^{\alpha}$ where $0<\alpha<1$; in the latter case it matters whether $\alpha$ is rational or not.

The case of random graphs with a fixed bound on the maximum degree, but no other constraint, has received less attention than the cases discussed so far. However, papers by Kennedy and Quintas [9] and Rucinski and Wormald [14, 15] are exceptions from this neglect and study the set of graphs with vertices $1, \ldots, n$ such that every vertex has degree at most $R$, which we denote $\mathbf{G}_{n, R}$. They do this from the point of view of a random process which generates members of $\mathbf{G}_{n, R}$ by randomly adding new edges as long as it is possible without violating the condition that the maximum degree is $R$. Kennedy and Quintas [9] stresses the importance of graphs with degree limitations from the perspective of models of physical and chemical systems and gives several references to work concerned with these aspects. Rucinski and Wormald [14] prove that with probability approaching 1 as $n$ tends to infinity, the random process ends up with a graph with $\lfloor n R / 2\rfloor$ edges. In [15] they prove that if $R=2$, then the number of $k$-cycles $(k \geq 3)$ in a graph produced by the random process has a Poisson distribution.

However, the main motivation of this article for studying $\mathbf{G}_{n, R}$ comes from logic and from understanding the fine structure of $\mathcal{H}$-free graphs for certain types of $\mathcal{H}$. From the point of view of model theory in logic, a restriction on the maximum degree is natural and can be generalised to arbitrary relational structures via the so-called Gaifman graph (e.g. [6]) of the structure. Finite relational structures and firstorder logic correspond closely to relational databases and the standard query language SQL, respectively (e.g. [11], Chapter 1.1). Within the subfield of model theory which studies logical limit laws one may be interested in better understanding which classes of structures (e.g. graphs) have a logical limit (or even zero-one) law and which do not. When a logical limit law exists for a given logical language and collection $\mathbf{C}$ of finite structures it follows that, for every property $P$ which can be expressed in that language, the proportion of structures which satisfy $P$ stabilises as the number of elements in the structure tends to infinity. Such information may be of help when understanding the average performance of algorithms that take random members of $\mathbf{C}$ as input.

Another type of restriction on graphs, studied in graph theory and interesting from a model theoretic point of view, is to forbid the occurrence of subgraphs which are isomorphic to some specified graph $\mathcal{H}$ (or to a member of a specified set of graphs). The results of this paper are relevant for understanding $\mathcal{H}$-free graphs for certain types of graphs $\mathcal{H}$. Let us call a vertex $v$ of the graph $\mathcal{H}$ colour critical if the removal of $v$ from $\mathcal{H}$ leaves a graph with lower chromatic number than $\mathcal{H}$. The criticality of $v$ is the minimal number of edges containing $v$ which have to be removed in order to reduce the chromatic number. Hundack, Prömel and Steger [8] have proved that if $\mathcal{H}$ is a graph with chromatic number $l+1(l \geq 2)$ and a colour critical vertex $v$ of criticality $R+1$ and $\operatorname{Forb}_{n}(\mathcal{H})$ is the set of $\mathcal{H}$-free graphs with vertices $1, \ldots, n$, then the proportion of $\mathcal{G} \in \operatorname{Forb}_{n}(\mathcal{H})$ which have the following property approaches 1 as $n \rightarrow \infty$ : the vertex set can be partitioned into parts $V_{1}, \ldots, V_{l}$ such that for every $i$ the induced subgraph with vertex set $V_{i}$ has maximum degree $R$. Hence, understanding of the asymptotic fine structure of graphs with maximum degree $R$ helps us to understand the asymptotic fine structure of $\mathcal{H}$-free graphs, for certain $\mathcal{H}$. This connection is used in [10] to prove a logical limit law (but not zero-one law) for $\mathcal{H}$-free graphs, when 
$\mathcal{H}$ is a complete $(l+1)$-partite graph $(l \geq 2)$ in which one part is a singleton.

If nothing else is said then we assume that $R \geq 2$ is an integer. We always consider the uniform probability distribution on $\mathbf{G}_{n, R}$. In Section 2 we study the typical degree distribution of a random $\mathcal{G} \in \mathbf{G}_{n, R}$ as $n \rightarrow \infty$. We find that, as $n \rightarrow \infty$, a random $\mathcal{G} \in \mathbf{G}_{n, R}$ almost surely has no vertices with degree less than $R-2$ and, for every $\varepsilon>0$, the number of vertices with degree $R-1$ is almost surely between $\sqrt{(R-\varepsilon) n}$ and $\sqrt{(R+\varepsilon) n}$, where almost surely means that the probability of the event approaches 1 as $n \rightarrow \infty$. The number of vertices with degree $R-2$ has, asymptotically, a Poisson distribution. These results are summarised in Theorem 2.1. To prove it we consider multigraphs and the configuration model of Bollobás [3, 4].

Section 3 deals with the typical structure of members of $\mathbf{G}_{n, R}$ as $n \rightarrow \infty$. The results are summarised in Theorem 3.1. Intuitively speaking, there are three types of "rare" events, which are vertices with degree $R-2$, short cycles and short paths with endpoints of degree $R-1$. They are, asymptotically, Poisson distributed and independent of each other. Moreover, these rare events typically occur far from each other. For example, the distance between any vertex with degree $R-2$ and any path of length 3 (say) with both endpoints of degree $R-1$ will almost surely be large. Also, the "semi-rare" event of being a vertex with degree $R-1$ almost surely occurs far from any rare event. The proofs use the configuration model of Bollobás [3, 4] and the fact, given by Theorem 2.1, that we can restrict attention to degree sequences with certain properties.

In Section 4, we use Theorems 2.1 and 3.1 to prove a labelled logical limit law for $\mathbf{G}_{n, R}$. In the cases $R=0$ and $R=1$ the result is known (and trivial), in both the labelled and unlabelled context, so we need only consider the case $R \geq 2$. If $R \geq 5$ then we can also conclude that $\mathbf{G}_{n, R}$ satisfies an unlabelled limit law, by applying a result of McKay and Wormald [13], saying that in both the labelled and unlabelled cases a random member of $\mathbf{G}_{n, R}$ almost surely has no non-trivial automorphism, and a general model theoretic result concerning labelled and unlabelled probabilities. We cannot apply the main result of [12] to get the limit laws of this paper, because the degree sequences that are typical of members of $\mathbf{G} \in \mathbf{G}_{n, R}$ do not satisfy condition (1)(b) of [12]. Moreover, the structural results of Sections 2 and 3 are needed for understanding logical limit laws of $\mathcal{H}$-free graphs in [10].

Preliminaries, terminology and notation. See for example [6, 11, 16] for definitions of first-order logic, By the language of graphs we mean the first-order formulas which can be built from the vocabulary (also called signature) which consists of a binary relation symbol $E$ (denoting the edge relation) and the identity symbol ' $=$ '. More generally, a finite relational vocabulary consists of a list of relation symbols $R_{1}, \ldots, R_{k}$ (and we can assume that $R_{1}$ is '=') each with an associated arity. A structure for the language associated with this vocabulary is a tuple $\mathcal{S}=\left(S, R_{1}^{\mathcal{S}}, \ldots, R_{1}^{\mathcal{S}}\right)$ such that $S$ is a set and $R_{i}^{\mathcal{S}} \subseteq S^{r_{i}}$ where $r_{i}$ is the arity of $R_{i}$. From this point of view a graph is a structure for the language of graphs, so it has the form $\mathcal{G}=\left(V, E^{\mathcal{G}}\right)$ where $(a, b) \in E^{\mathcal{G}}$ implies $(b, a) \in E^{\mathcal{G}}$ since we consider undirected graphs here. Alternatively we may, as in graph theory, view $E^{\mathcal{G}}$ as the set of edges (2-subsets of $V$ ) of $\mathcal{G}$. For a first-order sentence (a formula with no free variables) $\varphi$ in the language of graphs and a graph $\mathcal{G}, \mathcal{G}=\varphi$ is shorthand for " $\mathcal{G}$ satisfies $\varphi$ ". See for example [6, 11] for a formal definition of ' $=$ '.

The degree of a vertex $v$ in a graph $\mathcal{G}$ is denoted $\operatorname{deg}_{\mathcal{G}}(v)$, and the notation $v \sim_{\mathcal{G}} w$ means that vertices $v$ and $w$ are adjacent in $\mathcal{G}$ (which is equivalent with $\mathcal{G} \models E(v, w)$ ). By $\operatorname{dist}_{\mathcal{G}}(v, w)$ we mean the distance from $v$ to $w$ in $\mathcal{G}$. For functions $f$ and $g$ we say that $f$ is asymptotic with $g$, denoted $f \sim g$, if $\lim _{x \rightarrow \infty} f(x) / g(x)=1$. For positive integers $n$ we sometimes use the notation $[n]=\{1, \ldots, n\}$. To make notation simpler we sometimes omit explicit use of the floor function \lfloor\rfloor . For a set $S,|S|$ denotes 
its cardinality, $\mathbb{N}_{0}$ is the set of non-negative integers.

\section{Distribution of degrees}

For positive integers $n$ and $R, \mathbf{G}_{n, R}$ denotes the set of graphs with vertices $1, \ldots, n$ in which the degree of every vertex is at most $R$.

Theorem 2.1 Suppose that $R \geq 2$.

(i) The proportion of $\mathcal{G} \in \mathbf{G}_{n, R}$ which have no vertex with degree less than $R-2$ approaches 1 as $n \rightarrow \infty$.

(ii) For every $\varepsilon>0$, the proportion of $\mathcal{G} \in \mathbf{G}_{n, R}$ which have between $\sqrt{(R-\varepsilon) n}$ and $\sqrt{(R+\varepsilon) n}$ vertices with degree $R-1$ approaches 1 as $n \rightarrow \infty$.

(iii) For every $k \in \mathbb{N}_{0}$, the proportion of $\mathcal{G} \in \mathbf{G}_{n, R}$ which have exactly $k$ vertices with degree $R-2$ approaches

$$
\frac{(R-1)^{k} e^{-(R-1)}}{k !} \quad \text { as } n \rightarrow \infty .
$$

In other words, the number of vertices with degree $R-2$ has, asymptotically, a Poisson distribution with mean $R-1$.

If $3 \leq r \leq R$ and $\bar{d}=\left(d_{r}, \ldots, d_{R}\right)$ is a sequence of non-negative integers summing to $n$, then let $\mathbf{G}_{n, \bar{d}}$ be the set of all graphs $\mathcal{G}$ with vertices $1, \ldots, n$ such that, for $i=r, \ldots, R, \mathcal{G}$ has exactly $d_{i}$ vertices with degree $i$. Wormald (Theorem 1 in [17]) and McKay and Wormald (Corollaries 3.8 and 3.10 in [13]) have proved the following:

Theorem 2.2 ([17, 13]) If $3 \leq r \leq R$ and $\bar{d}=\left(d_{r}, \ldots, d_{R}\right)$ is a sequence of non-negative integers summing to $n$, then

(i) the number of r-connected $\mathcal{G} \in \mathbf{G}_{n, \bar{d}}$ is $\left|\mathbf{G}_{n, \bar{d}}\right|\left(1-O\left(n^{2-r}\right)\right)$ where $O()$ denotes a bound depending only on $R$, and

(ii) the expected number of nontrivial automorphisms of a random $\mathcal{G} \in \mathbf{G}_{n, \bar{d}}$ is $O\left(n^{2-r}\right)$ where $O($ ) denotes a bound depending only on $R$.

Statement (ii) is true also if we count graphs in $\mathbf{G}_{n, \bar{d}}$ only up to isomorphism, or in other words, if we consider unlabelled graphs with $n$ vertices and degree sequence $\bar{d}$.

Let us call a graph rigid if it has only one automorphism, the trivial one. Theorem 4.3.4 in [6], if stated in the context of graphs, says the following.

Theorem 2.3 ([6]) For every positive integer $n$, let $\mathbf{H}_{n} \subseteq \mathbf{G}_{n}$ be sets of graphs with vertices $1, \ldots, n$. Suppose that, for every $n$, if $\mathcal{G} \in \mathbf{H}_{n}, \mathcal{H}$ has vertices $1, \ldots, n$ and is isomorphic to $\mathcal{G}$, then $\mathcal{H} \in \mathbf{H}_{n}$; and if $\mathcal{G} \in \mathbf{G}_{n}, \mathcal{H}$ has vertices $1, \ldots, n$ and is isomorphic to $\mathcal{G}$, then $\mathcal{H} \in \mathbf{G}_{n}$. For every $n$ and $\mathcal{G} \in \mathbf{G}_{n}$, let 
$[\mathcal{G}]$ denote the equivalence class to which $\mathcal{G}$ belongs with respect to the isomorphism relation on $\mathbf{G}_{n}$. Let $\mathbf{R I G}\left(\mathbf{G}_{n}\right)$ be the set of $\mathcal{G} \in \mathbf{G}_{n}$ which are rigid.

$$
\text { If } \begin{aligned}
\lim _{n \rightarrow \infty} \frac{\left|\left\{[\mathcal{G}]: \mathcal{G} \in \mathbf{R I G}\left(\mathbf{G}_{n}\right)\right\}\right|}{\left|\left\{[\mathcal{G}]: \mathcal{G} \in \mathbf{G}_{n}\right\}\right|}=1 \text { then } \\
\lim _{n \rightarrow \infty} \frac{\left|\left\{[\mathcal{G}]: \mathcal{G} \in \mathbf{H}_{n}\right\}\right|}{\left|\left\{[\mathcal{G}]: \mathcal{G} \in \mathbf{G}_{n}\right\}\right|}=\lim _{n \rightarrow \infty} \frac{\left|\mathbf{H}_{n}\right|}{\left|\mathbf{G}_{n}\right|},
\end{aligned}
$$

if any one of the last two limits exist.

We say that a graph has connectivity $k$ if it is $k$-connected but not $(k+1)$-connected. (By $k$-connected we mean $k$-vertex connected.) By combining Theorems 2.1, 2.2 and 2.3 we get the following information about typical members of $\mathbf{G}_{n, R}$ for large $n$.

Corollary 2.4 Suppose that $R \geq 5$. The proportion of graphs $\mathcal{G} \in \mathbf{G}_{n, R}$ (or of isomorphism classes $[\mathcal{G}]$ where $\mathcal{G} \in \mathbf{G}_{n, R}$ ) which have the following two properties approaches 1 as $n$ approaches infinity:

(i) The connectivity of $\mathcal{G}$ is $R-2$ or $R-1$.

(ii) $\mathcal{G}$ is rigid.

Corollary 2.4 will be used to derive an unlabelled logical limit law in Section 4.

\subsection{Multigraphs and configurations}

Theorem 2.1 will be proved via the use of multigraphs and so-called configurations (defined below). The corresponding statements (of Theorem 2.1) for multigraphs are given by Lemmas 2.11, 2.12 and 2.13 below. In Section 2.2 we explain how Theorem 2.1 follows from these lemmas and from asymptotic estimates, of Bollobás [3, 4], of probabilities of small cycles in configurations. We fix an arbitrary integer $R \geq 1$.

Definition 2.5 A multigraph is an object of the form $(V, f)$ where $f: U \rightarrow\{0,1,2, \ldots\}$ and $U=$ $\{W \subseteq V: 1 \leq|W| \leq 2\}$. If $\mathcal{M}=(V, f)$ is a multigraph and $v \in V$ then we define $\operatorname{deg}_{\mathcal{M}}(v)=$ $2 f(\{v\})+\sum_{\substack{w \in V \\ w \neq v}} f(\{v, w\})$ and call $\operatorname{deg}_{\mathcal{M}}(v)$ the degree of $v$ (in $\left.\mathcal{M}\right)$.

Suppose that $(V, f)$ is a multigraph, $\{v, w\} \in U=\{W \subseteq V: 1 \leq|W| \leq 2\}$ and $f(\{v, w\})=k$. Intuitively this means that there are exactly $k$ different edges between $v$ and $w$. If $v=w$ then it means that there are exactly $k$ different loops which begin and end in $v$. If $f(W) \leq 1$ for all $W \in U$ and $f(W)=0$ whenever $|W|=1$, then $(V, f)$ corresponds to the graph $(V, E)$ where, for all distinct $v, w \in V,\{v, w\} \in E$ if and only if $f(\{v, w\})=1$.

\section{Definition 2.6}

(i) Let $\mathbf{M G}_{n, R}$ be the set of multigraphs with vertices $1, \ldots, n$ such that every vertex has degree at most $R$.

(ii) For every sequence $\bar{d}=\left(d_{0}, \ldots, d_{R}\right)$ of non-negative integers such that $\sum_{i=0}^{R} d_{i}=n$ we let $\mathbf{M G}_{n, \bar{d}}$ denote the set of multigraphs with vertices $1, \ldots, n$ such that there are exactly $d_{i}$ vertices with degree $i$ for $i=0, \ldots, R$. 
(iii) Let $N\left(d_{0}, \ldots, d_{R}\right)=\left|\mathbf{M G}_{n, \bar{d}}\right|$.

Definition 2.7 Let $E$ and $F$ be binary relation symbols and let $L_{c}$ be the first-order language with vocabulary $\{=, E, F\}$. A configuration is an $L_{c}$-structure $\mathcal{C}=\left(C, E^{\mathcal{C}}, F^{\mathcal{C}}\right)$ such that $F^{\mathcal{C}}$ is an equivalence relation on $C$ and $E^{\mathcal{C}}$ is an equivalence relation on $C$ such that every $E^{\mathcal{C}}$-class has cardinality 2 . The later condition implies that $|C|$ must be even (or infinite). An $E^{\mathcal{C}}$-class of a configuration $\mathcal{C}$ will often be called an edge (of $\mathcal{C}$ ). We say that $\mathcal{C}=\left(C, E^{\mathcal{C}}, F^{\mathcal{C}}\right)$ is an $n$-configuration if $F^{\mathcal{C}}$ has exactly $n$ nonempty classes.

Observe that if $\mathcal{C}=\left(C, E^{\mathcal{C}}, F^{\mathcal{C}}\right)$ is a configuration, then $\left(C, E^{\mathcal{C}}\right)$ is a graph which is a complete matching (also called perfect matching), i.e. every $c \in C$ is adjacent to exactly one member of $C$.

Definition 2.8 If $m>0$ is an integer then let $\mathrm{M}(2 m)$ denote the number of complete matchings, i.e. equivalence relations such that every class has cardinality 2 , on a set with cardinality $2 m$.

It is easy to see that

$$
\mathrm{M}(2 m)=\left(\begin{array}{c}
2 m \\
2
\end{array}\right)\left(\begin{array}{c}
2 m-2 \\
2
\end{array}\right) \cdots\left(\begin{array}{l}
2 \\
2
\end{array}\right) / m !=\frac{(2 m) !}{m ! 2^{m}} .
$$

By Stirling's approximation,

$$
\frac{(2 m) !}{m ! 2^{m}}=\left(\frac{2 m}{e}\right)^{m}(\sqrt{2}+o(1))
$$

Also note that for any fixed integer $p$ we have $m ! /(m-p) ! \sim m^{p}$ which gives

$$
\frac{\mathrm{M}(2 m)}{\mathrm{M}(2 m-2 p)}=\frac{(2 m) !}{m ! 2^{m}} / \frac{(2(m-p)) !}{(m-p) ! 2^{m-p}} \sim(2 m)^{p} .
$$

Definition 2.9 We will always assume that $W_{1}, \ldots, W_{n}$ is a sequence of disjoint sets such that $\sum_{i=1}^{n}\left|W_{i}\right|=2 m$ for some integer $m>0$. By $\mathbf{C}\left(W_{1}, \ldots, W_{n}\right)$ we denote the set of configurations $\mathcal{C}=\left(C, E^{\mathcal{C}}, F^{\mathcal{C}}\right)$ such that $C=\bigcup_{i=1}^{n} W_{i}$ and $W_{1}, \ldots, W_{n}$ enumerates all the $F^{\mathcal{C}}$-classes (so if some set $W_{i}$ other than an $F^{\mathcal{C}}$-class appears in the sequence then $W_{i}$ is empty).

Observe that if $2 m=\sum_{i=1}^{n}\left|W_{i}\right|$, then

$$
\left|\mathbf{C}\left(W_{1}, \ldots, W_{n}\right)\right|=\mathrm{M}(2 m)=\frac{(2 m) !}{m ! 2^{m}} .
$$

Definition 2.10 Let $\mathcal{C} \in \mathbf{C}\left(W_{1}, \ldots, W_{n}\right)$. The multigraph image of $\mathcal{C}$, denoted $\operatorname{Graph}(\mathcal{C})$, is the multigraph with vertices $1, \ldots, n$ such that, for all $i, j \in\{1, \ldots, n\}$, the number of edges between $i$ and $j$ is the same as the number of $E^{\mathcal{C}}$-classes which have non-empty intersection with both $W_{i}$ and $W_{j}$. ( $E^{\mathcal{C}}$-classes which are included in a single $W_{i}$, if they exist, give rise to loops in $\operatorname{Graph}(\mathcal{C})$.)

Observe that for every $\mathcal{C} \in \mathbf{C}\left(W_{1}, \ldots, W_{n}\right)$ and every $i=1, \ldots, n$, the degree of $i$ in $\operatorname{Graph}(\mathcal{C})$ is $\left|W_{i}\right|$. Moreover, if $\mathcal{G}$ is a multigraph with vertices $1, \ldots, n$ such that, for $i=1, \ldots, n, i$ has degree $\left|W_{i}\right|$, then there are exactly $\prod_{i=1}^{n}\left|W_{i}\right| !$ configurations $\mathcal{C} \in \mathbf{C}\left(W_{1}, \ldots, W_{n}\right)$ such that $\operatorname{Graph}(\mathcal{C})=\mathcal{G}$, because $\operatorname{Graph}\left(\mathcal{C}^{\prime}\right)=\operatorname{Graph}(\mathcal{C})$ if and only if there is an isomorphism from $\mathcal{C}^{\prime}$ to $\mathcal{C}$ which preserves every $W_{i}$ 
setwise. It follows that if $\bar{d}=\left(d_{0}, \ldots, d_{R}\right)$ is a sequence of non-negative integers such that $\sum_{i=0}^{R} d_{i}=n$ and $\sum_{i=0}^{R} i d_{i}=2 m$, then, using $[3$, we have

$$
N\left(d_{0}, \ldots, d_{R}\right)=\left(\begin{array}{c}
n \\
d_{0}, \ldots, d_{R}
\end{array}\right) \frac{(2 m) !}{m ! 2^{m}} / \prod_{i=0}^{R}(i !)^{d_{i}} .
$$

From now on assume that $\bar{d}=\left(d_{0}, \ldots, d_{R}\right)$ is a sequence of non-negative integers such that $\sum_{i=0}^{R} d_{i}=$ $n$ and $\sum_{i=0}^{R} i d_{i}=2 m$ for some integer $m>0$. We are interested in the probability that a random $\mathcal{G} \in \mathbf{M G}_{n, R}$ (drawn uniformly) has this degree sequence. In other words, we consider the proportion

$$
p\left(d_{0}, \ldots, d_{R}\right)=\frac{N\left(d_{0}, \ldots, d_{R}\right)}{\left|\mathbf{M G}_{n, R}\right|}=\frac{N\left(d_{0}, \ldots, d_{R}\right)}{\sum N\left(d_{0}^{\prime}, \ldots, d_{R}^{\prime}\right)},
$$

where the sum ranges over all sequences $\left(d_{0}^{\prime}, \ldots, d_{R}^{\prime}\right)$ of non-negative integers such that $\sum_{i=0}^{R} d_{i}^{\prime}=n$.

If $n$ is even and all vertices have degree $R$, that is, if $d_{i}=0$ for $i=0, \ldots, R-1$, we get $2 m=R n$ and, by (4) and (1),

$$
N(0, \ldots, 0, n)=\left(\frac{R n}{e}\right)^{R n / 2} \frac{1}{(R !)^{n}}(\sqrt{2}+o(1)) \geq C_{1}^{n} n^{R n / 2}
$$

for some constant $C_{1}>0$. If $n$ is odd, then, with $d_{0}=1, d_{i}=0$ for $i=1, \ldots, R-1$ and $d_{R}=n-1$, we have $2 m=R(n-1)$ and get in a similar way

$$
N(1,0, \ldots, 0, n-1) \geq C_{2}^{n} n^{R n / 2}
$$

for some constant $C_{2}>0$.

Let $\varepsilon>0$. We now estimate the proportion of $\mathcal{G} \in \mathbf{M G}_{n, R}$ for which $2 m \leq(R-\varepsilon) n$. Summing over all $\left(d_{0}, \ldots, d_{R}\right)$ for which $\sum_{i=0}^{R} i d_{i}=2 m \leq(R-\varepsilon) n$ gives, for large enough $n$ and using $(5)$ and $(6)$,

$$
\begin{aligned}
& \sum_{2 m \leq(R-\varepsilon) n} N\left(d_{0}, \ldots, d_{R}\right) \leq \sum_{2 m \leq(R-\varepsilon) n}\left(\begin{array}{c}
n \\
d_{0}, \ldots, d_{R}
\end{array}\right) \frac{(2 m) !}{m ! 2^{m}} / \prod_{i=0}^{R}(i !)^{d_{i}} \\
\leq & (R+1)^{n}\left(\frac{(R-\varepsilon) n}{e}\right)^{(R-\varepsilon) n / 2} \cdot 2=2(R+1)^{n}\left(\frac{(R-\varepsilon) n}{e}\right)^{R n / 2}\left(\frac{(R-\varepsilon) n}{e}\right)^{-\varepsilon n / 2} \\
\leq & \left(C_{3} n^{-\varepsilon / 2}\right)^{n} N, \text { where } N=N(0, \ldots, 0, n) \text { or } N=N(1,0, \ldots, 0, n-1) \\
& \text { depending on whether } n \text { is even or odd and } C_{3}>0 \text { is a constant. }
\end{aligned}
$$

It follows that

$$
\frac{\sum_{2 m \leq(R-\varepsilon) n} N\left(d_{0}, \ldots, d_{R}\right)}{\left|\mathbf{M G}_{n, R}\right|} \leq\left(C_{3} n^{-\varepsilon / 2}\right)^{n} \rightarrow 0 \quad \text { as } n \rightarrow \infty .
$$

Therefore we will assume that $2 m>(R-\varepsilon) n$. Since $\varepsilon>0$ is arbitrary we may in fact assume that $2 m>(R-o(1)) n$. Since

$$
R n-2 m=R n-\sum_{i=0}^{R} i d_{i}=\sum_{i=0}^{R}(R-i) d_{i}
$$


and $2 m>(R-o(1)) n$ now implies $R n-2 m \leq o(n)$ we get

$$
\frac{\sum_{i=0}^{R}(R-i) d_{i}}{n} \leq o(1)
$$

which in turn implies that

$$
d_{i}=o(n) \text { for all } i=0, \ldots, R-1 .
$$

Next, we aim at showing that in the typical case we have $d_{i}=0$ for all $i=0, \ldots, R-3$. So until further notice we assume that $R \geq 3$ since otherwise there is nothing to prove. Fix any $i \leq R-3$ and suppose that $d_{i} \geq 1$. Now let

$$
\begin{aligned}
& d_{i}^{\prime}=d_{i}-1, \\
& d_{i+2}^{\prime}=d_{i+2}+1 \text { and } \\
& d_{j}^{\prime}=d_{j} \text { for all } j \notin\{i, i+2\} .
\end{aligned}
$$

With $2 m^{\prime}=\sum_{j=0}^{R} j d_{j}^{\prime}$ we have $2 m^{\prime}=2 m+2$ (where $2 m=\sum_{j=0}^{R} j d_{j}$ ). Now we get, by 4 , and 2 ,

$$
\begin{aligned}
& \frac{N\left(d_{0}, \ldots, d_{R}\right)}{N\left(d_{0}^{\prime}, \ldots, d_{R}^{\prime}\right)} \\
= & \left(\begin{array}{c}
n \\
d_{0}, \ldots, d_{R}
\end{array}\right) \frac{(2 m) !}{m ! 2^{m}}\left(\prod_{j=0}^{R}(j !)^{d_{j}}\right)^{-1} /\left(\begin{array}{c}
n \\
d_{0}^{\prime}, \ldots, d_{R}^{\prime}
\end{array}\right) \frac{\left(2 m^{\prime}\right) !}{m^{\prime} ! 2^{m^{\prime}}}\left(\prod_{j=0}^{R}(j !)^{d_{j}^{\prime}}\right)^{-1} \\
\sim & \frac{d_{i}^{\prime} ! d_{i+2}^{\prime} !}{d_{i} ! d_{i+2} !} \frac{1}{2 m} \prod_{j=0}^{R}(j !)^{d_{j}^{\prime}-d_{j}}=\frac{d_{i+2}+1}{d_{i}} \frac{1}{2 m}(i+2)(i+1) .
\end{aligned}
$$

As we assume that $2 m>(R-o(1)) n$ we may assume that $2 m \geq n$. If $i \leq R-3$ then, by (7), $d_{i+2}=o(n)$ so we may assume that $d_{i+2}+1 \leq f(n)$ where $f(n)=o(n)$, which gives

$$
\frac{N\left(d_{0}, \ldots, d_{R}\right)}{N\left(d_{0}^{\prime}, \ldots, d_{R}^{\prime}\right)} \leq \frac{f(n) R^{2}}{n}
$$

Hence, for $i \leq R-3$,

$$
p\left(d_{0}, \ldots, d_{R}\right) \leq \frac{R^{2} f(n)}{n} p\left(d_{0}^{\prime}, \ldots, d_{R}^{\prime}\right),
$$

and if we sum over all $\left(d_{0}, \ldots, d_{R}\right)$ for which $d_{i} \geq 1, d_{i+2} \leq f(n)$ and $2 m \geq n$, we get

$$
\begin{aligned}
\sum p\left(d_{0}, \ldots, d_{R}\right) & \leq \frac{R^{2} f(n)}{n} \sum p\left(d_{0}^{\prime}, \ldots, d_{R}^{\prime}\right) \\
& \leq \frac{R^{2} f(n)}{n}=o(1) \quad(\text { since } f(n)=o(n)) .
\end{aligned}
$$

This implies that the proportion of $\mathcal{G} \in \mathbf{M G}_{n, R}$ which have a vertex with degree less than $R-2$ approaches 0 as $n \rightarrow \infty$. Therefore we assume, from now on, that

$$
d_{i}=0 \text { for all } i=0, \ldots, R-3 .
$$

We also state this conclusion as: 
Lemma 2.11 The proportion of $\mathcal{G} \in \mathbf{M G}_{n, R}$ such that $\mathcal{G}$ has a vertex with degree less than $R-2$ approaches 0 as $n \rightarrow \infty$.

The next step is to find a lower upper bound (than in (7) for $d_{R-2}$ in the typical case, so we assume that $R \geq 2$ until further notice. Suppose that $g(n) \rightarrow \infty$ as $n \rightarrow \infty$ and suppose that $d_{R-2} \geq g(n)$. We argue as before, with $i=R-2$, so $d_{R-2}^{\prime}=d_{R-2}-1, d_{R}^{\prime}=d_{R}+1$ and $d_{j}^{\prime}=d_{j}$ if $j \notin\{R-2, R\}$. Just as before, and as $2 m>(R-o(1)) n$ where $R \geq 2$, we get

$$
\frac{N\left(d_{0}, \ldots, d_{R}\right)}{N\left(d_{0}^{\prime}, \ldots, d_{R}^{\prime}\right)} \sim \frac{d_{R}+1}{d_{R-2}} \frac{1}{2 m} R(R-1)<\frac{R^{2}}{d_{R-2}} \leq \frac{R^{2}}{g(n)} .
$$

Hence $p\left(d_{0}, \ldots, d_{R}\right) \leq R^{2} p\left(d_{0}^{\prime}, \ldots, d_{R}^{\prime}\right) / g(n)$ and if we sum over all $\left(d_{0}, \ldots, d_{R}\right)$ such that $d_{R-2} \geq$ $g(n)$ we get

$$
\sum p\left(d_{0}, \ldots, d_{R}\right) \leq \frac{R^{2}}{g(n)}
$$

Therefore we may assume that

$$
d_{R-2} \leq g(n) \text { for every } g \text { such that } \lim _{n \rightarrow \infty} g(n)=\infty .
$$

The next step is to show that in the typical case $d_{R-1}=O(\sqrt{n})$. Assume that $R \geq 1$ and that $d_{R-1} \geq 2$. Let

$$
\begin{aligned}
& d_{R-1}^{\prime}=d_{R-1}-2, \\
& d_{R}^{\prime}=d_{R}+2 \text { and } \\
& d_{j}^{\prime}=d_{j} \text { if } j \notin\{R-1, R\} .
\end{aligned}
$$

Then $2 m^{\prime}=2 m+2$ where $2 m^{\prime}=\sum_{j=0}^{R} i d_{j}^{\prime}$ and

$$
\begin{aligned}
\frac{N\left(d_{0}, \ldots, d_{R}\right)}{N\left(d_{0}^{\prime}, \ldots, d_{R}^{\prime}\right)} & \sim \frac{\left(d_{R}+1\right)\left(d_{R}+2\right)}{d_{R-1}\left(d_{R-1}-1\right)} \frac{1}{2 m} R^{2} \\
& \leq \frac{\left(d_{R}+1\right) n}{d_{R-1}\left(d_{R-1}-1\right)} \frac{1}{R d_{R}} R^{2} \leq \frac{4 R n}{d_{R-2}^{2}}
\end{aligned}
$$

It follows that

$$
p\left(d_{0}, \ldots, d_{R}\right) \leq \frac{4 R n}{d_{R-1}^{2}} p\left(d_{0}^{\prime}, \ldots, d_{R}^{\prime}\right) .
$$

Let $\mathbb{P}\left(d_{R-1} \geq k\right)$ denote the proportion of $\mathcal{G} \in \mathbf{M G}_{n, R}$ which have at least $k$ vertices with degree $R-1$. Then for every $k \geq 2$, by summation over all $\left(d_{0}, \ldots, d_{R}\right)$ such that $d_{R-1} \geq k$, we get

$$
\mathbb{P}\left(d_{R-1} \geq k\right) \leq \frac{4 R n}{k^{2}} \mathbb{P}\left(d_{R-1} \geq k-2\right) .
$$

If $C>2 \sqrt{R}$ then, by iterating $\sqrt{10} \sqrt{n}$ times, we get that the proportion of $\mathcal{G} \in \mathbf{M G}_{n, R}$ which have at least $(C+2)\lceil\sqrt{n}\rceil$ vertices with degree $R-1$ is

$$
\mathbb{P}\left(d_{R-1} \geq(C+2)\lceil\sqrt{n}\rceil\right) \leq\left(\frac{4 R n}{(C \sqrt{n})^{2}}\right)^{\sqrt{n}}=\left(\frac{4 R}{C^{2}}\right)^{\sqrt{n}} \rightarrow 0 \quad \text { as } n \rightarrow \infty .
$$


Therefore we may assume, in addition to the previous assumptions (8) and (9), that

$$
d_{R-1}=O(\sqrt{n}) .
$$

We use the notation $(n)_{k}=n(n-1) \cdots(n-k+1)$. By equation (3.8) in [1], for example, we have:

$$
\text { If } k=O(\sqrt{n}) \text {, then }(n)_{k}=n^{k} \exp \left(-\frac{k^{2}}{2 n}+o(1)\right) \text {. }
$$

To make the argument here self contained we prove $\sqrt{12})$. If $k=O(\sqrt{n})$ then

$$
\begin{aligned}
\ln \left(\frac{(n)_{k}}{n^{k}}\right) & =\ln \frac{n !}{(n-k) ! n^{k}}=\ln \left(\prod_{i=0}^{k-1} \frac{n-i}{n}\right) \\
& =\ln \left(\prod_{i=0}^{k-1}\left(1-\frac{i}{n}\right)\right)=\sum_{i=0}^{k-1} \ln \left(1-\frac{i}{n}\right),
\end{aligned}
$$

and for large $n$ and $i<k=O(\sqrt{n}), i / n$ is close to 0 , so by Taylor's approximation we get

$$
\begin{aligned}
\sum_{i=0}^{k-1} \ln \left(1-\frac{i}{n}\right) & =\sum_{i=0}^{k-1}\left(-\frac{i}{n}+O\left(\left(\frac{i}{n}\right)^{2}\right)\right) \\
& =-\frac{k(k-1)}{2 n}+O\left(\frac{k^{3}}{n^{2}}\right)=-\frac{k^{2}}{2 n}+\frac{k}{2 n}+O\left(\frac{k^{3}}{n^{2}}\right) \\
& =-\frac{k^{2}}{2 n}+o(1), \quad \text { since } k=O(\sqrt{n}) .
\end{aligned}
$$

Hence, $\ln \left(\frac{(n)_{k}}{n^{k}}\right)=-\frac{k^{2}}{2 n}+o(1)$ which immediately gives 12 .

Recall the exact formula (4) for $N\left(d_{0}, \ldots, d_{R}\right)$ and remember the assumptions (8), (9) and (11). Hence $d_{i}=0$ for $i=0, \ldots, R-3, d_{R-2}=o(\sqrt{n}), d_{R-1}=O(\sqrt{n}), d_{R}=n-d_{R-1}-d_{R-2}$ and $2 m=R n-d_{R-1}-2 d_{R-2}$ (since, by definition, $2 m=\sum_{i=0}^{R} i d_{i}$ ). By also using 12 we get

$$
\begin{aligned}
& N\left(d_{0}, \ldots, d_{R}\right)= \\
& =\left(\begin{array}{c}
n \\
d_{R-2}, d_{R-1}, d_{R}
\end{array}\right) \cdot \frac{(2 m) !}{m ! 2^{m}} \cdot \frac{1}{((R-2) !)^{d_{R-2}}((R-1) !)^{d_{R-1}}(R !)^{d_{R}}} \\
& =\frac{(n)_{d_{R-2}+d_{R-1}}}{d_{R-2} ! d_{R-1} !} \cdot \frac{(2 m) !}{m ! 2^{m}} \cdot \frac{(R(R-1))^{d_{R-2}} R^{d_{R-1}}}{(R !)^{n}} \\
& =\frac{n^{d_{R-2}+d_{R-1}} \exp \left(-\frac{\left(d_{R-2}+d_{R-1}\right)^{2}}{2 n}+o(1)\right)}{d_{R-2} ! d_{R-1} !} \cdot \frac{(2 m) !}{m ! 2^{m}} \cdot \frac{(R(R-1))^{d_{R-2}} R^{d_{R-1}}}{(R !)^{n}} \text {. }
\end{aligned}
$$

From $2 m=R n-d_{R-1}-2 d_{R-2}$ and 12 it follows that

$$
\frac{(2 m) !}{m ! 2^{m}}=\frac{(R n) !}{\lfloor R n / 2\rfloor ! 2^{R n / 2}} \cdot \frac{\exp \left(\frac{\left(d_{R-1}+2 d_{R-2}\right)^{2}}{4 R n}+o(1)\right)}{(R n)^{\left(d_{R-1}+2 d_{R-2}\right) / 2}} .
$$


By combining (13) and (14) we get

$$
\begin{aligned}
N\left(d_{0}, \ldots, d_{R}\right)= & C_{n} \cdot \frac{(R-1)^{d_{R-2}}}{d_{R-2} !} \cdot \frac{(\sqrt{R n})^{d_{R-1}}}{d_{R-1} !} \\
& \exp \left(-\frac{\left(d_{R-1}+d_{R-2}\right)^{2}}{2 n}+\frac{\left(d_{R-1}+2 d_{R-2}\right)^{2}}{4 R n}+o(1)\right),
\end{aligned}
$$

where

$$
C_{n}=\frac{(R n) !}{\lfloor R n / 2\rfloor ! 2^{R n / 2}} \cdot \frac{1}{(R !)^{n}}
$$

depends only on $n$. Note that since $d_{R-2}=o(\sqrt{n})$ and $d_{R-1}=O(\sqrt{n})$ it follows that the expression ' $\exp (\ldots)$ ' in 15 is bounded as $n \rightarrow \infty$.

Lemma 2.12 (i) For every $c<R$ the proportion of $\mathcal{G} \in \mathbf{M G}_{n, R}$ with less than $\sqrt{c n}$ vertices with degree $R-1$ approaches 0 as $n \rightarrow \infty$.

(ii) For every $c>R$ the proportion of $\mathcal{G} \in \mathbf{M G}_{n, R}$ with more than $\sqrt{c n}$ vertices with degree $R-1$ approaches 0 as $n \rightarrow \infty$.

Proof: (ii) Let $c<R$. Since there are not more than $n$ possibilities for $d_{R-1}$, it suffices to prove that for all large enough $n$,

$$
\frac{N\left(0, \ldots, 0, d_{R-2},\lfloor\sqrt{c n}\rfloor, d_{R}\right)}{N\left(0, \ldots, 0, d_{R-2},\lfloor\sqrt{c n}\rfloor+2\left\lfloor n^{1 / 4}\right\rfloor, d_{R}\right)}=\left(\sqrt{\frac{c}{R}}+o(1)\right)^{2\left\lfloor n^{1 / 4}\right\rfloor} \cdot O(1) .
$$

We may assume that $d_{R-2}=o(\sqrt{n})$ so by $\sqrt{12}$ and $\sqrt{15}$ the above quotient equals

$$
\begin{aligned}
& (\sqrt{R n})^{-2\left\lfloor n^{1 / 4}\right\rfloor} \cdot \frac{\left(\lfloor\sqrt{c n}\rfloor+2\left\lfloor n^{1 / 4}\right\rfloor\right) !}{\lfloor\sqrt{c n}\rfloor !} \cdot O(1)=(\sqrt{R n})^{-2\left\lfloor n^{1 / 4}\right\rfloor} \cdot\left(\lfloor\sqrt{c n}\rfloor+2\left\lfloor n^{1 / 4}\right\rfloor\right)_{2\left\lfloor n^{1 / 4}\right\rfloor} \\
= & (\sqrt{R n})^{-2\left\lfloor n^{1 / 4}\right\rfloor}\left(\lfloor\sqrt{c n}\rfloor+2\left\lfloor n^{1 / 4}\right\rfloor\right)^{2\left\lfloor n^{1 / 4}\right\rfloor} \cdot O(1)=\left(\frac{\lfloor\sqrt{c n}\rfloor+2\left\lfloor n^{1 / 4}\right\rfloor}{\sqrt{R n}}\right)^{2\left\lfloor n^{1 / 4}\right\rfloor} \cdot O(1) \\
= & \left(\sqrt{\frac{c}{R}}+o(1)\right)^{2\left\lfloor n^{1 / 4}\right\rfloor} \cdot O(1) .
\end{aligned}
$$

Part (ii), where $c>R$, is proved in a similar way by considering the quotient

$$
\frac{N\left(0, \ldots, 0, d_{R-2},\lfloor\sqrt{c n}\rfloor, d_{R}\right)}{N\left(0, \ldots, 0, d_{R-2},\lfloor\sqrt{c n}\rfloor-2\left\lfloor n^{1 / 4}\right\rfloor, d_{R}\right)} .
$$

Lemma 2.13 For every fixed $d_{R-2}$, the proportion of $\mathcal{G} \in \mathbf{M G}_{n, R}$ which have exactly $d_{R-2}$ vertices with degree $R-2$ approaches

$$
\frac{(R-1)^{d_{R-2}} e^{-(R-1)}}{d_{R-2} !} .
$$


Proof: Let $d_{R-2}$ be fixed and let $c>R$. We know, by (9) and Lemma 2.12, that the proportion of $\mathcal{G} \in \mathbf{M G}_{n, R}$ such that $\mathcal{G}$ has more than $\left\lfloor n^{1 / 4}\right\rfloor$ vertices with degree $R-2$ or more than $\sqrt{c n}$ vertices with degree $R-1$, approaches 0 as $n \rightarrow \infty$. Therefore it suffices to prove that whenever $d_{R-1} \leq \sqrt{c n}$,

$$
\frac{N\left(0, \ldots, 0, d_{R-2}, d_{R-1}, d_{R}\right)}{\sum_{k=0}^{\left\lfloor n^{1 / 4}\right\rfloor} N\left(0, \ldots, 0, k, d_{R-1}, d_{R}\right)}=(1 \pm o(1)) \frac{(R-1)^{d_{R-2}} e^{-(R-1)}}{d_{R-2} !} .
$$

By $[15$, the above quotient equals

$$
(1 \pm o(1)) \frac{\frac{(R-1)^{d} R-2}{d_{R-2} !}}{\sum_{k=0}^{\left\lfloor n^{1 / 4}\right\rfloor} \frac{(R-1)^{k}}{k !}}=(1 \pm o(1)) \frac{\frac{(R-1)^{d_{R-2}}}{d_{R-2} !}}{e^{R-1}}=(1 \pm o(1)) \frac{(R-1)^{d_{R-2}} e^{-(R-1)}}{d_{R-2} !} .
$$

\subsection{Configurations and graphs}

In this section we explain why Lemmas 2.11, 2.12 and 2.13 also apply to graphs without multiple edges or loops.

Definition 2.14 Let $\mathcal{C}=\left(C, E^{\mathcal{C}}, F^{\mathcal{C}}\right)$ be a configuration and let $p \geq 1$ be an integer. By a path (of $\mathcal{C}$ ) from $a \in C$ to $b \in C$ of length $p$, or $p$-path from $a$ to $b$, we mean sequence of $p$ distinct edges (i.e. $E^{\mathcal{C}}$-classes) $e_{1}, \ldots, e_{p}$ such that there are $F^{\mathcal{C}}$-classes $W_{1}, \ldots, W_{p+1}$ such $a \in e_{1} \cap W_{1}, b \in e_{p} \cap W_{p+1}, W_{i} \neq W_{j}$ if $i \neq j$ and $i \neq 1$ or if $i \neq j$ and $j \neq p+1$ (but we allow that $W_{1}=W_{p+1}$ ) and, for $i=1, \ldots, p, e_{i}$ contains a member of $W_{i}$ and a member of $W_{i+1}$. In the described situation we call $a$ and $b$ the endpoints of the path. By a p-cycle (of $\mathcal{C}$ ) we mean a $p$-path from $a$ to $b$ for some $a$ and $b$ such that $(a, b) \in F^{\mathcal{C}}$; or in other words, a $p$-path from $a$ to $b$ where $a, b \in W$ for some $F^{\mathcal{C}}$-class $W$.

Recall Definition 2.9 of $\mathbf{C}\left(W_{1}, \ldots, W_{n}\right)$ and Definition 2.10 about the multigraph image $\operatorname{Graph}(\mathcal{C})$ of a configuration $\mathcal{C}$. It is easy to see that that if $\mathcal{C} \in \mathbf{C}\left(W_{1}, \ldots, W_{n}\right)$ and $\mathcal{C}$ has no $p$-cycle for $p=1,2$, then $\operatorname{Graph}(\mathcal{C})$ has no loops or multiple edges.

Definition 2.15 By $\mathbf{C}^{\prime}\left(W_{1}, \ldots, W_{n}\right)$ we denote the set of configurations $\mathcal{C} \in \mathbf{C}\left(W_{1}, \ldots, W_{n}\right)$ which have no $p$-cycle for $p=1,2$.

The proof of Theorem 1 in [3] (or Theorem 2.16 in [4]) shows the following:

Fact 2.16 $([3,4])$ Let $W_{1}, \ldots, W_{n}$ be disjoint sets and for $i=1, \ldots, R$, let

$$
d_{i}=\left|\left\{j:\left|W_{j}\right|=i\right\}\right| .
$$

Suppose that for all $i<R-2, d_{i}=0$ and $d_{R-2}+d_{R-1}=O(\sqrt{n})$.

(i) Then

$$
\left|\frac{\left|\mathbf{C}^{\prime}\left(W_{1}, \ldots, W_{n}\right)\right|}{\left|\mathbf{C}\left(W_{1}, \ldots, W_{n}\right)\right|}-\exp \left(-\frac{R-1}{2}-\frac{(R-1)^{2}}{4}\right)\right|=o(1) \quad \text { as } n \rightarrow \infty,
$$

where the bound ' $o($ )' depends only on $R$. 
(ii) From (3) and (4) it follows that if we let $\bar{d}=\left(d_{0}, \ldots, d_{R}\right)$ (with $d_{i}$ as above for $i=1, \ldots, R$ and $\left.d_{0}=0\right)$, then

$$
\left|\frac{\left|\mathbf{G}_{n, \bar{d}}\right|}{\left|\mathbf{M G}_{n, \bar{d}}\right|}-\exp \left(-\frac{R-1}{2}-\frac{(R-1)^{2}}{4}\right)\right|=o(1) \quad \text { as } n \rightarrow \infty,
$$

where the bound ' $o($ )' depends only on $R$.

From part (ii) of the fact it immediately follows that Lemmas 2.11 and 2.12 apply to graphs as well, that is, they remain true if we replace $\mathbf{M} \mathbf{G}_{n, R}$ by $\mathbf{G}_{n, R}$. To see why Lemma 2.13 also holds with $\mathbf{G}_{n, R}$ in the place of $\mathbf{M G}_{n, R}$ consider its proof. There we showed that

$$
\frac{N\left(0, \ldots, 0, d_{R-2}, d_{R-1}, d_{R}\right)}{\sum_{k=0}^{\left\lfloor n^{1 / 4}\right\rfloor} N\left(0, \ldots, 0, k, d_{R-1}, d_{R}\right)}=(1 \pm o(1)) \frac{(R-1)^{d_{R-2}} e^{-(R-1)}}{d_{R-2} !} .
$$

By Fact 2.16 (ii), if $\bar{d}=\left(0, \ldots, 0, d_{R-2}, d_{R-1}, d_{R}\right), d_{R-2}+d_{R-1}=O(\sqrt{n})$, then

$$
\left|\mathbf{G}_{n, \bar{d}}\right|=(1 \pm o(1)) \exp \left(-\frac{R-1}{2}-\frac{(R-1)^{2}}{4}\right) N\left(0, \ldots, 0, d_{R-2}, d_{R-1}, d_{R}\right) .
$$

In the same way, for each $\bar{d}^{\prime}=\left(0, \ldots, 0, k, d_{R-1}, d_{R}\right)$, we also get (assuming $k+d_{R-1}=O(\sqrt{n})$ )

$$
\left|\mathbf{G}_{n, \bar{d}^{\prime}}\right|=(1 \pm o(1)) \exp \left(-\frac{R-1}{2}-\frac{(R-1)^{2}}{4}\right) N\left(0, \ldots, 0, k, d_{R-1}, d_{R}-k\right)
$$

Therefore, the proof of Lemma 2.13 implies that, for any fixed $d_{R-2}$, the proportion of $\mathcal{G} \in \mathbf{G}_{n, R}$ such that $\mathcal{G}$ has exactly $d_{R-2}$ vertices with degree $R-2$ approaches

$(R-1)^{d_{R-2}} e^{-(R-1)} / d_{R-2}$ ! as $n \rightarrow \infty$. In other words, Lemma 2.13 holds if $\mathbf{M G} \mathbf{G}_{n, R}$ is replaced by $\mathbf{G}_{n, R}$. This completes the proof of Theorem 2.1

\section{The typical asymptotic structure}

In this section we study the typical structure of large graphs with maximum degree $R$, where $R \geq 2$ is a fixed integer. The following theorem summarises the results that will be proved.

Theorem 3.1 Let $R \geq 2$ be an integer.

(i) For every integer $k>0$ the proportion of graphs $\mathcal{G} \in \mathbf{G}_{n, R}$ which have properties (a)-(d) below approaches 1 as $n \rightarrow \infty$ :

(a) If $p \leq k$ then $\mathcal{G}$ has no subgraph $\mathcal{H}$ with exactly $p$ vertices and more than $p$ edges. It follows that whenever $p_{1}, p_{2} \geq 3$ and $p_{1}+p_{2}+p_{3} \leq k$, then $\mathcal{G}$ does not have a $p_{1}$-cycle and a $p_{2}$-cycle such that there is a $p_{3}$-path from a vertex in the first cycle to a vertex in the second cycle.

(b) If $p, q \leq k$ then there is no vertex $v$ with degree less than $R$ and p-path from $v$ to a vertex that belongs to a q-cycle. In particular, no q-cycle contains a vertex of degree less than $R$. 
(c) There do not exist distinct vertices $v_{1}, v_{2}, v_{3}$ all of which have degree at most $R-1$ such that for all distinct $i, j \in\{1,2,3\}$ there is a path of length at most $k$ from $v_{i}$ to $v_{j}$.

(d) There do not exist distinct vertices $v$ and $w$ such that $\operatorname{deg}_{\mathcal{G}}(v) \leq R-1, \operatorname{deg}_{\mathcal{G}}(w) \leq R-2$ and there is a path of length at most $k$ from $v$ to $w$.

(ii) Let $k \geq 3$ be an integer. There are positive $\lambda_{3}, \ldots, \lambda_{k}, \mu_{1}, \ldots, \mu_{k} \in \mathbb{Q}$ such that for all $r_{3}, \ldots, r_{k}$, $s_{1}, \ldots, s_{k} \in \mathbb{N}_{0}$ the proportion of $\mathcal{G} \in \mathbf{G}_{n, R}$ which, for $p=3, \ldots, k$, have exactly $r_{p} p$-cycles and, for $p=1, \ldots, k$, have exactly $s_{p}$ p-paths with both endpoints of degree $R-1$, approaches

$$
\left(\prod_{p=3}^{k} \frac{\left(\lambda_{p}\right)^{r_{p}} e^{-\lambda_{p}}}{r_{p} !}\right)\left(\prod_{p=1}^{k} \frac{\left(\mu_{p}\right)^{s_{p}} e^{-\mu_{p}}}{s_{p} !}\right) \text { as } n \rightarrow \infty .
$$

Moreover, the mentioned proportion approaches this value independently of the number of vertices with degree $R-2$.

(iii) If $R \geq 3$ then for every $k \in \mathbb{N}_{0}$ the proportion of $\mathcal{G} \in \mathbf{G}_{n, R}$ such that $\mathcal{G}$ has a connected component with at most $k$ vertices approaches 0 as $n \rightarrow \infty$.

Note that part (iii) of the theorem states that if $X_{p}$ is the number of $p$-cycles, for $p=3, \ldots, k$, and $Y_{p}$ is the number of $p$-paths with endpoints of degree $R-1$, for $p=1, \ldots, k$, then the random variables $X_{3}, \ldots, X_{p}, Y_{1}, \ldots, Y_{p}$ are, asymptotically, independent Poisson variables with means $\lambda_{3}, \ldots, \lambda_{k}$, $\mu_{1}, \ldots, \mu_{k}$, respectively, which are described in detail by Lemma 3.7 If one omits the consideration of short paths with endpoints of degree $R-1$ in part (iii), then the resulting statement is a straightforward consequence of Theorem 2.1 and either one of Theorem 1 in [3], (the proof of) Theorem 2.16 in [4], or Corollary 1 in [18]. However, knowing the distribution of short paths with endpoints of degree $R-1$ is necessary in the proof of the limit law in Section 4

\subsection{Graphs with a given degree sequence}

Fix an integer $R \geq 2$. By Theorem 2.1.

- the proportion of $\mathcal{G} \in \mathbf{G}_{n, R}$ without vertices with degree less than $R-2$ approaches 1 as $n \rightarrow \infty$,

- the number of vertices of $\mathcal{G} \in \mathbf{G}_{n, R}$ with degree $R-2$ has a Poisson distribution, asymptotically, and

- for every $\varepsilon>0$, the proportion of $\mathcal{G} \in \mathbf{G}_{n, R}$ which have between $\sqrt{(R-\varepsilon) n}$ and $\sqrt{(R+\varepsilon) n}$ vertices with degree $R-1$ approaches 1 as $n \rightarrow \infty$.

Therefore, there are $\varepsilon_{n}$ and $\delta_{n}$ such that $\lim _{n \rightarrow \infty} \varepsilon_{n}=\lim _{n \rightarrow \infty} \delta_{n}=0$, the proportion of $\mathcal{G} \in \mathbf{G}_{n, R}$ without vertices of degree 0 , with at most $n^{1 / 4}$ vertices with degree $R-2$ and with between $\sqrt{\left(R-\delta_{n}\right) n}$ and $\sqrt{\left(R+\delta_{n}\right) n}$ vertices with degree $R-1$ is at least $1-\varepsilon_{n}$. Let $\mathbf{G}_{n, R}^{\prime}$ be the set of all $\mathcal{G} \in \mathbf{G}_{n, R}$ such that $\mathcal{G}$ has no vertices of degree less than $R-2$, at most $n^{1 / 4}$ vertices of degree $R-2$ and between $\sqrt{\left(R-\delta_{n}\right) n}$ and $\sqrt{\left(R+\delta_{n}\right) n}$ vertices with degree $R-1$. It follows that $\left|\mathbf{G}_{n, R}^{\prime}\right| /\left|\mathbf{G}_{n, R}\right| \rightarrow 1$ as $n \rightarrow \infty$.

Since the number of edges of $\mathcal{G}=\left(V, E^{\mathcal{G}}\right)$ equals $\frac{1}{2} \sum_{v \in V} \operatorname{deg}_{\mathcal{G}}(v)$ it follows that $\sum_{v \in V} \operatorname{deg}_{\mathcal{G}}(v)$ must be even. For every positive $n \in \mathbb{N}_{0}$ and sequence of integers $\bar{d}=\left(d_{1}, d_{2}, \ldots, d_{n}\right)$ such that 
$0 \leq d_{i} \leq R$ for all $i=1, \ldots, n$ and $\sum_{i=1}^{n} d_{i}$ is even, let $\mathbf{G}_{n, R}(\bar{d})$ be the set of graphs $\mathcal{G}$ with vertices $1, \ldots, n$ such that $\operatorname{deg}_{\mathcal{G}}(i)=d_{i}$ for $i=1, \ldots, n .\left(\mathbf{G}_{n, R}(\bar{d})\right.$ is different from $\mathbf{G}_{n, \bar{d}}$ in Section 2$)$ ) A priori we do not know if $\mathbf{G}_{n, R}(\bar{d})$ is non-empty for every $\bar{d}$ such that $\sum_{i=1}^{n} d_{i}$ is even. But for such degree sequences $\bar{d}$ as we will consider (satisfying $(16)$ below) it follows from Fact 2.16 above that $\mathbf{G}_{n, R}(\bar{d})$ is indeed non-empty if, in addition, $\sum_{i=1}^{n} d_{i}$ is even and $n$ is sufficiently large. Bollobás [3, 4] gives asymptotic estimates of $\left|\mathbf{G}_{n, R}(\bar{d})\right|$.

Now suppose that $\sigma_{n}$ are positive numbers such that $\lim _{n \rightarrow \infty} \sigma_{n}=0$, that $P$ is a property of graphs and that $0<c<1$. Moreover, assume that whenever $n$ is large enough and $\bar{d}=\left(d_{1}, d_{2}, \ldots, d_{n}\right)$ satisfies that

$$
\begin{aligned}
& R-2 \leq d_{i} \leq R \text { for all } i=1, \ldots, n,\left|\left\{i: d_{i}=R-2\right\}\right| \leq n^{1 / 4} \text { and } \\
& \sqrt{\left(R-\delta_{n}\right) n} \leq\left|\left\{i: d_{i}=R-1\right\}\right| \leq \sqrt{\left(R+\delta_{n}\right) n}
\end{aligned}
$$

and $\mathbf{G}_{n, R}(\bar{d}) \neq \emptyset$, then the proportion of $\mathcal{G} \in \mathbf{G}_{n, R}(\bar{d})$ which have property $P$ is at least $c-\sigma_{n}$ and at most $c+\sigma_{n}$. If $\mathbf{P}_{n}$ is the set of all $\mathcal{G} \in \mathbf{G}_{n, R}^{\prime}$ which have property $P$ and, for every $\bar{d}$ satisfying $\sqrt{16}$, $\mathbf{P}_{n}(\bar{d})$ is the set of $\mathcal{G} \in \mathbf{G}_{n, R}(\bar{d})$ which have property $P$, then we get

$$
\begin{aligned}
& \left|\mathbf{P}_{n}\right|=\sum_{\bar{d}}\left|\mathbf{P}_{n}(\bar{d})\right| \geq\left(c-\sigma_{n}\right) \sum_{\bar{d}}\left|\mathbf{G}_{n, R}(\bar{d})\right|=\left(c-\sigma_{n}\right)\left|\mathbf{G}_{n, R}^{\prime}\right| \\
& \text { and in a similar way }\left|\mathbf{P}_{n}\right| \leq\left(c+\sigma_{n}\right)\left|\mathbf{G}_{n, R}^{\prime}\right|,
\end{aligned}
$$

where the sums range over all $\bar{d}$ which satisfy $(16)$, so $c-\sigma_{n} \leq\left|\mathbf{P}_{n}\right| /\left|\mathbf{G}_{n, R}^{\prime}\right| \leq c+\sigma_{n}$. It follows that the proportion of $\mathcal{G} \in \mathbf{G}_{n, R}^{\prime}$ which have property $P$ approaches $c$ as $n \rightarrow \infty$ and hence also the proportion of $\mathcal{G} \in \mathbf{G}_{n, R}$ which have property $P$ approaches $c$ as $n \rightarrow \infty$. To summarise the argument, we have:

Conclusion. To prove that the proportion of $\mathcal{G} \in \mathbf{G}_{n, R}$ which have a property $P$ approaches c as $n \rightarrow \infty$, independently of the number of vertices with degree $R-2$ in $\mathcal{G}$, it suffices to prove that for every degree sequence $\bar{d}$ which satisfies $(16)$ and such that $\sum_{i=1}^{n} d_{i}$ is even, the proportion of $\mathcal{G} \in \mathbf{G}_{n, R}(\bar{d})$ which has $P,\left|\mathbf{P}_{n}(\bar{d})\right| /\left|\mathbf{G}_{n, R}(\bar{d})\right|$, approaches $c$ as $n \rightarrow \infty$ and || $\mathbf{P}_{n}(\bar{d})|/| \mathbf{G}_{n, R}(\bar{d})|-c|$ is bounded by a function which tends to zero and depends only on $P$ and $R$.

\subsection{Proof of Theorem 3.1}

The lemmas of this section prove the different parts of Theorem 3.1. Let $R \geq 2$ be an integer. Until the proof of Lemma 3.7 is finished, we assume that $W_{1}, \ldots, W_{n}$ are disjoint sets, $d_{i}=\left|W_{i}\right|, \sum_{i=1}^{n} d_{i}=2 m$ and that $(16)$ holds. This implies that

$$
2 m \sim R n .
$$

As we observed in Sections 2.1 and 2.2 if $\bar{d}=\left(d_{1}, \ldots, d_{n}\right)$, then $\mathbf{G}_{n, R}(\bar{d})$ can be identified with

$$
\left\{\operatorname{Graph}(\mathcal{C}): \mathcal{C} \in \mathbf{C}^{\prime}\left(W_{1}, \ldots, W_{n}\right)\right\} .
$$

By Fact 2.16 and the discussion in Section 3.1. it follows that in order to prove that the proportion of $\mathcal{G} \in \mathbf{G}_{n, R}$ with property $P$ approaches $c$ as $n \rightarrow \infty$, it now suffices to prove that

$$
\left|\frac{\mid\left\{\mathcal{C} \in \mathbf{C}^{\prime}\left(W_{1}, \ldots, W_{n}\right): \operatorname{Graph}(\mathcal{C}) \text { has } P\right\} \mid}{\left|\mathbf{C}^{\prime}\left(W_{1}, \ldots, W_{n}\right)\right|}-c\right|=o(1) \quad \text { as } n \rightarrow \infty,
$$

where the bound $o($ ) depends only on $P$ and $R$. 
Lemma 3.2 For all integers $p \geq 1$ and $q \geq 3$, the proportion of $\mathcal{G} \in \mathbf{G}_{n, R}$ which have a vertex $v$ of degree less than $R$ and a p-path from $v$ to a vertex in a q-cycle approaches 0 as $n \rightarrow \infty$. It follows that the proportion of $\mathcal{G} \in \mathbf{G}_{n, R}$ which have a vertex $v$ of degree less than $R$ such that $v$ belongs to a $q$-cycle approaches 0 as $n \rightarrow \infty$.

Proof: Let $p \geq 1$ and $q \geq 3$. If $\mathcal{C} \in \mathbf{C}^{\prime}\left(W_{1}, \ldots, W_{n}\right)$ and $\operatorname{Graph}(\mathcal{C})$ has a vertex $W_{i}$ with degree less than $R$ and a $p$-path from $W_{i}$ to a vertex in a $q$-cycle, then $\left|W_{i}\right|<R$ and there are $W_{j}$, a $p$-path $e_{1}, \ldots, e_{p}$ $\left(\right.$ of $\mathcal{C}$ ) and a $q$-cycle $e_{1}^{\prime}, \ldots, e_{q}^{\prime}$ (of $\mathcal{C}$ ) such that $e_{1} \cap W_{i} \neq \emptyset, e_{p} \cap W_{j} \neq \emptyset$ and $e_{1}^{\prime} \cap W_{j} \neq \emptyset$. If $W_{i}$ belongs to a $q$-cycle of $\operatorname{Graph}(\mathcal{C})$, then let $W_{j}=W_{i}$ and note that a $p$-path as $e_{1}, \ldots, e_{p}$ of $\mathcal{C}$ as above need not exist, but the argument below (with $p=0$ and finding at most $q-1$ other $F$-classes than $W_{i}$ ) shows that the corresponding quotient (below) still approaches 0 as $n \rightarrow \infty$; this will prove the second statement of the lemma. Therefore it suffices to prove that the proportion of $\mathcal{C} \in \mathbf{C}\left(W_{1}, \ldots, W_{n}\right)$ such that for some $W_{i}$ with $\left|W_{i}\right|<R$ there are $W_{j}$, a $p$-path $e_{1}, \ldots, e_{p}$ and a $q$-cycle $e_{1}^{\prime}, \ldots, e_{q}^{\prime}$ such that $e_{1} \cap W_{i} \neq \emptyset$, $e_{p} \cap W_{j} \neq \emptyset$ and $e_{1}^{\prime} \cap W_{j} \neq \emptyset$ approaches 0 as $n \rightarrow \infty$.

By assumption $\sqrt{16}$ we can choose $W_{i}$ with cardinality less than $R$ in at most $n^{1 / 4}+\sqrt{(R+\delta(n)) n}$ ways, where $\delta(n) \rightarrow 0$ as $n \rightarrow \infty$. A $p$-path $e_{1}, \ldots, e_{p}$ and a $q$-cycle $e_{1}^{\prime}, \ldots, e_{q}^{\prime}$ such that $e_{p} \cap W_{j} \neq \emptyset$ and $e_{1}^{\prime} \cap W_{j} \neq \emptyset$ for some $j$ can intersect at most $p-1+q$ different $F$-classes other than $W_{i}$. Hence the at most $p+q-1 F$-classes other than $W_{i}$ which are going to include the union of the $p$-path and $q$-cycle as above can be chosen in at most $n^{p+q-1}$ ways. Then the $2(p+q)$ elements which are going to form the union of the edges $e_{1}, \ldots, e_{p}, e_{1}^{\prime}, \ldots, e_{q}^{\prime}$ can be chosen in no more than $((p+q) R)^{2(p+q)}$ ways. Then a complete matching on these $2(p+q)$ elements (that is, non-intersecting edges $\left.e_{1}, \ldots, e_{p}, e_{1}^{\prime}, \ldots, e_{q}^{\prime}\right)$ can be chosen in $\mathrm{M}(2(p+q))$ ways. Finally, a complete matching on the remaining $2 m-(2 p+2 q)$ elements can be chosen in $\mathrm{M}(2 m-(2 p+2 q))$ ways. Therefore, there is a constant $\alpha>0$ depending only on $p$, $q$ and $R$ such that, for all sufficiently large $n$, the proportion of $\mathcal{C} \in \mathbf{C}\left(W_{1}, \ldots, W_{n}\right)$ for which there are $W_{i}$ with $\left|W_{i}\right|<R, W_{j}$, a $p$-path $e_{1}, \ldots, e_{p}$ and a $q$-cycle $e_{1}^{\prime}, \ldots, e_{q}^{\prime}$ such that $e_{1} \cap W_{i} \neq \emptyset, e_{p} \cap W_{j} \neq \emptyset$ and $e_{1}^{\prime} \cap W_{j} \neq \emptyset$ is at most

$$
\begin{aligned}
\frac{\alpha \sqrt{n} n^{p+q-1} \mathrm{M}(2 m-2(p+q))}{\mathrm{M}(2 m)} & \sim \frac{\alpha n^{p+q-1 / 2}}{(2 m)^{p+q}} \sim \frac{\alpha n^{p+q-1 / 2}}{(R n)^{p+q}} \\
& =\frac{\alpha n^{-1 / 2}}{R^{p+q}} \rightarrow 0 \quad \text { as } n \rightarrow \infty
\end{aligned}
$$

where (2) was used in the first asymptotic identity.

The next lemma is a well known result in the case of $R$-regular graphs [19], and can be proved in a similar way in that case.

Lemma 3.3 If $\mathcal{H}$ is a graph with more edges than vertices, then the proportion of $\mathcal{G} \in \mathbf{G}_{n, R}$ which have a subgraph that is isomorphic to $\mathcal{H}$ approaches 0 as $n \rightarrow \infty$.

Proof: Let $\mathcal{H}$ be a graph with $p>0$ vertices and $q>p$ edges. If $\mathcal{C} \in \mathbf{C}\left(W_{1}, \ldots, W_{n}\right)$ (so in particular if $\left.\mathcal{C} \in \mathbf{C}^{\prime}\left(W_{1}, \ldots, W_{n}\right)\right)$ and $\operatorname{Graph}(\mathcal{C})$ has a subgraph $\mathcal{H}^{\prime}$ which is isomorphic to $\mathcal{H}$, then there are $W_{i_{1}}, \ldots, W_{i_{p}}$ and $p+1$ edges of $\mathcal{C}$ (i.e. $E$-classes) $e_{1}, \ldots, e_{p+1}$ such that $e_{j} \subseteq W_{i_{1}} \cup \ldots \cup W_{i_{p}}$ for $j=1, \ldots, p+1$. Hence it is sufficient to prove that the proportion of $\mathcal{C} \in \mathbf{C}\left(W_{1}, \ldots, W_{n}\right)$ such that there 
are $W_{i_{1}}, \ldots, W_{i_{p}}$ and $p+1$ edges of $\mathcal{C}, e_{1}, \ldots, e_{p+1}$, such that $e_{j} \subseteq W_{i_{1}} \cup \ldots \cup W_{i_{p}}$ for $j=1, \ldots, p+1$ approaches 0 as $n \rightarrow \infty$.

We can choose $W_{i_{1}}, \ldots, W_{i_{p}}$ in at most $n^{p}$ ways, and then choose $p+1$ disjoint 2-subsets of $\bigcup_{j=1}^{p} W_{i_{j}}$ in at most $\alpha=\left(\begin{array}{c}R p \\ 2\end{array}\right)\left(\begin{array}{c}R p-2 \\ 2\end{array}\right) \cdots\left(\begin{array}{c}R p-2 p \\ 2\end{array}\right)$ ways. The remaining $2 m-2(p+1)$ elements can be completely matched in $\mathrm{M}(2 m-2(p+1))$ ways. Consequently, the proportion of $\mathcal{C} \in \mathbf{C}\left(W_{1}, \ldots, W_{n}\right)$ such that there are $W_{i_{1}}, \ldots, W_{i_{p}}$ and $p+1$ edges of $\mathcal{C}, e_{1}, \ldots, e_{p+1}$, such that $e_{j} \subseteq W_{i_{1}} \cup \ldots \cup W_{i_{p}}$ for $j=1, \ldots, p+1$ is at most

$$
\frac{\alpha n^{p} \mathrm{M}(2 m-2(p+1))}{\mathrm{M}(2 m)} \sim \frac{\alpha n^{p}}{(2 m)^{p+1}} \sim \frac{\alpha n^{p}}{(R n)^{p+1}}=\frac{\alpha}{R^{p+1} n} \rightarrow 0 \text { as } n \rightarrow \infty .
$$

Remark 3.4 Observe that Lemma 3.3 implies the following: For all integers $p \geq 0$ and $q \geq 3$, the proportion of $\mathcal{G} \in \mathbf{G}_{n, R}$ for which there are $3 \leq i, j \leq q$, an $i$-cycle and a different $j$-cycle within distance $p$ of each other, approaches 0 as $n \rightarrow \infty$.

Lemma 3.5 For every integer $p>0$, the proportion of $\mathcal{G} \in \mathbf{G}_{n, R}$ that have three distinct vertices $v_{1}, v_{2}, v_{3}$ with degree less than $R$ such that for all distinct $i, j \in\{1,2,3\}$ there is a path of length at most p from $v_{i}$ to $v_{j}$, approaches 0 as $n \rightarrow \infty$.

Proof: Let $p$ be a positive integer. It suffices to prove that, for every choice of positive integers $p_{1}, p_{2} \leq p$, the proportion of $\mathcal{C} \in \mathbf{C}\left(W_{1}, \ldots, W_{n}\right)$ with the following property approaches 0 as $n \rightarrow \infty$ :

(*) There are $W_{i_{1}}, W_{i_{2}}, W_{i_{3}}$ with $\left|W_{i_{1}}\right|,\left|W_{i_{2}}\right|,\left|W_{i_{3}}\right|<R$ such that there is a $p_{1}$-path with endpoints in $W_{i_{3}}$ and $W_{i_{1}}$ and a $p_{2}$-path with endpoints in $W_{i_{3}}$ and $W_{i_{2}}$.

Let $c>R$. By Assumption $\sqrt{16}$, for all large enough $n$ there are at most $\sqrt{c n} F$-classes $W_{i}$ with cardinality less than $R$, so $W_{i_{1}}, W_{i_{2}}, W_{i_{3}}$ can be chosen in at most $(c n)^{3 / 2}$ ways. The other $p_{1}+p_{2}-2$ $F$-classes which the $p_{1}$-path and $p_{2}$-path are going to intersect can be chosen in at most $n^{p_{1}+p_{2}-2}$ ways. The union of all edges in the two paths will contain $2\left(p_{1}+p_{2}\right)$ elements, which can be chosen in no more than $\left(\left(p_{1}+p_{2}+1\right) R\right)^{2\left(p_{1}+p_{2}\right)}$ ways, since they belong to the already chosen $F$-classes. A complete matching (forming two paths) on these elements can be chosen in no more than $\mathrm{M}\left(2\left(p_{1}+p_{2}\right)\right)$ ways, and a complete matching on the remaining $2 m-2\left(p_{1}+p_{2}\right)$ elements can be chosen in $\mathrm{M}\left(2 m-2\left(p_{1}+p_{2}\right)\right)$ ways. Hence, for some constant $\alpha$ depending only on $p_{1}, p_{2}$ and $R$, the proportion of $\mathcal{C} \in \mathbf{C}\left(W_{1}, \ldots, W_{n}\right)$ that satisfy $(*)$ is at most

$$
\begin{aligned}
\frac{\alpha n^{3 / 2} n^{p_{1}+p_{2}-2} \mathrm{M}\left(2 m-2\left(p_{1}+p_{2}\right)\right)}{\mathrm{M}(2 m)} & \sim \frac{\alpha n^{p_{1}+p_{2}-1 / 2}}{(2 m)^{p_{1}+p_{2}}} \sim \frac{\alpha n^{p_{1}+p_{2}-1 / 2}}{(R n)^{p_{1}+p_{2}}} \\
& =\frac{\alpha n^{-1 / 2}}{R^{p_{1}+p_{2}}} \rightarrow 0 \quad \text { as } n \rightarrow \infty .
\end{aligned}
$$

Lemma 3.6 For every integer $p>0$, the proportion of $\mathcal{G} \in \mathbf{G}_{n, R}$ which have vertices $v$ and $w$ such that $\operatorname{deg}_{\mathcal{G}}(v) \leq R-2, \operatorname{deg}_{\mathcal{G}}(w) \leq R-1$ and a p-path from $v$ to $w$ approaches 0 as $n \rightarrow \infty$. 
Proof: Let $p>0$. It suffices to prove that the proportion of $\mathcal{C} \in \mathbf{C}\left(W_{1}, \ldots, W_{n}\right)$ which have $F$-classes $W_{i_{1}}, W_{i_{2}}$ such that $\left|W_{i_{1}}\right| \leq R-2$ and $\left|W_{i_{2}}\right| \leq R-1$ and a $p$-path starting in $W_{i_{1}}$ and ending in $W_{i_{2}}$ approaches 0 as $n \rightarrow \infty$. By Assumption $(16)$, one can choose $W_{i_{1}}$ and $W_{i_{2}}$ such that $\left|W_{i_{1}}\right| \leq R-2$ and $\left|W_{i_{2}}\right| \leq R-1$ in at most $n^{1 / 4} \cdot\left(\sqrt{(R+\delta(n)) n}+n^{1 / 4}\right)=\sqrt{R+\delta(n)} \cdot n^{3 / 4}+\sqrt{n}$ ways. The $p-1 F$ classes other than $W_{i_{1}}$ and $W_{i_{2}}$ which the $p$-path is going to intersect can be chosen in at most $n^{p-1}$ ways. The number of ways in which $2 p$ elements from $W_{i_{1}}, W_{i_{2}}$ and the other chosen $F$-classes can be chosen is bounded by a constant depending only on $p$ and $R$; the same is true for the number of ways of choosing a $p$-path from these elements. Finally, a complete matching on the remaining $2 m-2 p$ elements can be chosen in $\mathrm{M}(2 m-2 p)$ ways. So the proportion of $\mathcal{C} \in \mathbf{C}\left(W_{1}, \ldots, W_{n}\right)$ with the described property is, for some constant $\alpha$ depending only on $p$ and $R$, at most

$$
\frac{\alpha n^{3 / 4} n^{p-1} \mathrm{M}(2 m-2 p)}{\mathrm{M}(2 m)} \sim \frac{\alpha n^{p-1 / 4}}{(2 m)^{p}} \sim \frac{\alpha n^{p-1 / 4}}{(R n)^{p}}=\frac{\alpha n^{-1 / 4}}{R^{p}} \rightarrow 0 \quad \text { as } n \rightarrow \infty .
$$

Lemma 3.7 Let $k \geq 3$ be an integer and let $r_{3}, \ldots, r_{k}, s_{1}, \ldots, s_{k} \in \mathbb{N}_{0}$. Then the proportion of $\mathcal{G} \in$ $\mathbf{G}_{n, R}$ which, for $p=3, \ldots, k$, have exactly $r_{p}$ p-cycles and, for $p=1, \ldots, k$, have exactly $s_{p} p$-paths with both endpoints of degree $R-1$, approaches

$$
\left(\prod_{p=3}^{k} \frac{\left(\lambda_{p}\right)^{r_{p}} e^{-\lambda_{p}}}{r_{p} !}\right)\left(\prod_{p=1}^{k} \frac{\left(\mu_{p}\right)^{s_{p}} e^{-\mu_{p}}}{s_{p} !}\right) \text { as } n \rightarrow \infty,
$$

where $\lambda_{p}=\frac{(R-1)^{p}}{2 p}$ and $\mu_{p}=\frac{(R-1)^{p+1}}{2}$. In other words, if $X_{p}$ is the number of $p$-cycles and $Y_{p}$ is the number of p-paths with both endpoints of degree $R-1$, then the random variables $X_{p}, p=3, \ldots, k$ and $Y_{p}, p=1, \ldots, k$, are asymptotically independent Poisson variables with mean $\lambda_{p}$ and $\mu_{p}$, respectively. Moreover, the random variables $X_{p}, p=3, \ldots, k$, and $Y_{p}, p=1, \ldots, k$, are asymptotically independent of the (asymptotically Poisson distributed) number of vertices with degree $R-2$.

Proof: Let $k \geq 3$. The last statement of the lemma will follow from the argument below, because the only thing regarding the number of vertices with degree $R-2$, or in the context of configurations, the number of $F$-classes of cardinality $R-2$, is that this number is at most $n^{1 / 4}$; so the limit to be proved is independent of the number of vertices ( $F$-classes) with degree (cardinality) $R-2$. For $p=1, \ldots, k$, let the random variable $X_{p}$ be the number of $p$-cycles of a configuration $\mathcal{C} \in \mathbf{C}\left(W_{1}, \ldots, W_{n}\right)$ and let the random variable $Y_{p}$ be the number of $p$-paths ( of $\mathcal{C}$ ) with both endpoints in $F$-classes of cardinality $R-1$. For $p=1, \ldots, k$, let

$$
\lambda_{p}=\frac{(R-1)^{p}}{2 p} \quad \text { and } \quad \mu_{p}=\frac{(R-1)^{p+1}}{2} .
$$

We will prove:

Claim. $X_{1}, \ldots, X_{k}, Y_{1}, \ldots, Y_{k}$ are asymptotically independent Poisson variables with mean $\lambda_{1}, \ldots, \lambda_{k}$, $\mu_{1}, \ldots, \mu_{k}$, respectively.

By Fact 2.16 (ii), the probability that $X_{1}=X_{2}=0$ approaches $\exp \left(-\lambda_{1}-\lambda_{2}\right)$ as $n \rightarrow \infty$, and therefore (using part (iii) of the same fact) the lemma follows from the claim. Actually the argument that follows 
proves Fact 2.16 (ii), but also considers the random variables $Y_{1}, \ldots, Y_{k}$ of the lemma, which we must take into account when proving a logical limit law.

Recall the notation $(x)_{i}=x(x-1) \ldots(x-i+1)$. Note that the random variable

$$
Z=\left(X_{1}\right)_{r_{1}}\left(X_{2}\right)_{r_{2}} \ldots\left(X_{k}\right)_{r_{k}}\left(Y_{1}\right)_{s_{1}}\left(Y_{2}\right)_{s_{2}} \ldots\left(Y_{k}\right)_{s_{k}}
$$

is the number of ordered $2 k$-tuples without repetition consisting of (from left to right) $r_{1} 1$-cycles, $r_{2}$ 2-cycles, $\ldots, r_{k} k$-cycles, $s_{1} 1$-paths with both endpoints in $F$-classes of cardinality $R-1, \ldots$ and $s_{k}$ $k$-paths with both endpoints in $F$-classes of cardinality $R-1$. By Theorem 1.23 in [4], to prove the claim it suffices to prove that

$$
\left|\mathbb{E}(Z)-\prod_{p=1}^{k}\left(\lambda_{p}\right)^{r_{p}}\left(\mu_{p}\right)^{s_{p}}\right|=o(1) \quad \text { as } n \rightarrow \infty,
$$

where $\mathbb{E}()$ denotes the expected value and the bound ' $o()$ ' depends only on $k, R, r_{1}, \ldots, r_{k}$ and $s_{1}, \ldots, s_{k}$. Indeed, in the argument we never use any other properties of the degree sequence $\bar{d}=$ $\left(d_{1}, \ldots, d_{n}\right)$ than those stated in $(16)$, which hold for all degree sequences that we consider. Let $Z^{\prime}$ be the number of ordered $2 k$-tuples $\left(A_{1}, \ldots, A_{2 k}\right)$ without repetition as counted by $Z$ above, but with the extra condition that there is no $W_{i}$ such that for two distinct entries $A_{j}, A_{j^{\prime}}\left(j^{\prime} \neq j\right)$ and edges $e_{j} \in A_{j}$ and $e_{j^{\prime}} \in A_{j^{\prime}}, e_{j} \cap W_{i} \neq \emptyset$ and $e_{j^{\prime}} \cap W_{i} \neq \emptyset$. Let $Z^{\prime \prime}=Z-Z^{\prime}$, so $Z=Z^{\prime}+Z^{\prime \prime}$ and $\mathbb{E}(Z)=\mathbb{E}\left(Z^{\prime}\right)+\mathbb{E}\left(Z^{\prime \prime}\right)$. Let $C_{p}(n)$ be the number of ways to choose a $p$-cycle (a matching on a $2 p$-subset of $W_{1} \cup \ldots \cup W_{n}$ which forms a $p$-cycle), so we have

$$
C_{p}(n) \leq \frac{(R(R-1) n)^{p}}{2 p}
$$

Then let $P_{p}(n)$ be the number of ways to choose a $p$-path with both endpoints in $F$-classes with cardinality $R-1$, so by assumption (16),

$$
\begin{aligned}
P_{p}(n) & \leq \frac{(R-1)^{2}\left(R+\delta_{n}\right) n(R(R-1) n)^{p-1}}{2} \\
& \sim \frac{(R-1)(R(R-1) n)^{p}}{2} \text { as } n \rightarrow \infty .
\end{aligned}
$$

For every $A \subset \bigcup_{i=1}^{n} W_{i}$, let $C_{p}(n, A)$ and $P_{p}(n, A)$ be defined as $C_{p}(n)$ and $P_{p}(n)$ but with the extra condition that no chosen edge ( $E$-class) has non-empty intersection with $A$. Let $t=\sum_{p=1}^{k}\left(p r_{p}+p s_{p}\right)$ and let

$$
\begin{aligned}
& C_{p}(n, t)=\min \left\{C_{p}(n, A): A \subseteq \bigcup_{i=1}^{n} W_{i},|A|=2 t\right\}, \text { and } \\
& P_{p}(n, t)=\min \left\{C_{p}(n, A): A \subseteq \bigcup_{i=1}^{n} W_{i},|A|=2 t\right\} .
\end{aligned}
$$


Let $f(n)=n-\sqrt{\left(R+\delta_{n}\right) n}-n^{1 / 4}-2 t$. From $\sqrt{16}$ we get

$$
\begin{aligned}
C_{p}(n, t) & \geq \frac{(R(R-1) f(n))^{p}}{2 p} \sim \frac{(R(R-1) n)^{p}}{2 p} \quad \text { and } \\
P_{p}(n, t) & \geq \frac{(R-1)^{2}\left(\sqrt{\left(R-\delta_{n}\right) n}-2 t\right)^{2}}{2}(R(R-1) f(n))^{p-1} \\
& \sim \frac{(R-1)(R(R-1) n)^{p}}{2} \quad \text { as } n \rightarrow \infty .
\end{aligned}
$$

We have

$$
\prod_{p=1}^{k} C_{p}(n, t)^{r_{p}} P_{p}(n, t)^{s_{p}} \leq Z^{\prime} \leq \prod_{p=1}^{k} C_{p}(n)^{r_{p}} P_{p}(n)^{s_{p}},
$$

so by (18), (19), 20) and (21) we get

$$
Z^{\prime} \sim \prod_{p=1}^{k}\left(\frac{(R(R-1) n)^{p}}{2 p}\right)^{r_{p}}\left(\frac{(R-1)(R(R-1) n)^{p}}{2}\right)^{s_{p}} .
$$

For every tuple $\left(A_{1}, \ldots, A_{2 k}\right)$ that is being counted by $Z^{\prime}$, we have $\left|\bigcup_{p=1}^{2 k} A_{p}\right|=2 t$ and for every $A \subseteq \bigcup_{i=1}^{n} W_{i}$ with $|A|=2 t$ and every complete matching on $A$, the proportion of configurations in $\mathbf{C}\left(W_{1}, \ldots, W_{n}\right)$ which have this matching on $\mathrm{A}$ is

$$
\frac{\mathrm{M}(2 m-2 t)}{\mathrm{M}(2 m)} \sim(2 m)^{-t} \sim(R n)^{-t} .
$$

From this and 22, and recalling that $t=\sum_{p=1}^{k}\left(p r_{p}+p s_{p}\right)$, we get

$$
\begin{aligned}
\mathbb{E}\left(Z^{\prime}\right) & \sim(R n)^{-\sum_{p=1}^{k}\left(p r_{p}+p s_{p}\right)} \prod_{p=1}^{k}\left(\frac{(R(R-1) n)^{p}}{2 p}\right)^{r_{p}}\left(\frac{(R-1)(R(R-1) n)^{p}}{2}\right)^{s_{p}} \\
& =\prod_{p=1}^{k}\left(\frac{(R-1)^{p}}{2 p}\right)^{r_{p}}\left(\frac{(R-1)^{p+1}}{2}\right)^{s_{p}}=\prod_{p=1}^{k}\left(\lambda_{p}\right)^{r_{p}}\left(\mu_{p}\right)^{s_{p}} .
\end{aligned}
$$

By $18,, \sqrt{19}, 20$ and $21,\left|\mathbb{E}\left(Z^{\prime}\right)-\prod_{p=1}^{k}\left(\lambda_{p}\right)^{r_{p}}\left(\mu_{p}\right)^{s_{p}}\right|=o(1)$ as $n \rightarrow \infty$ where the bound depends only on $R, k, r_{1}, \ldots, r_{k}$ and $s_{1}, \ldots, s_{k}$.

Since $Z=Z^{\prime}+Z^{\prime \prime}$ it now suffices to prove that $\lim _{n \rightarrow \infty} \mathbb{E}\left(Z^{\prime \prime}\right)=o(1)$, where the bound depends only on $R, k, r_{1}, \ldots, r_{k}$ and $s_{1}, \ldots, s_{k}$, because this together with the conclusion above implies the statement of 17. But $Z^{\prime \prime}>0$ means that at least one of the following conditions holds:

(a) For some $q \leq t$ there are $W_{i_{1}}, \ldots, W_{i_{q}}$ and edges $e_{1}, \ldots, e_{q+1}$ such that $e_{j} \subseteq W_{i_{1}} \cup \ldots \cup W_{i_{q}}$ for $j=1, \ldots, q+1$.

(b) For some $q, q^{\prime} \leq t$ and some $W_{i}$ with $\left|W_{i}\right|<R$ there are $W_{j}$, a $q$-path $e_{1}, \ldots, e_{q}$ and $q^{\prime}$-cycle $e_{1}^{\prime}, \ldots, e_{q^{\prime}}^{\prime}$ such that $e_{1} \cap W_{i} \neq \emptyset, e_{q} \cap W_{j} \neq \emptyset$ and $e_{1}^{\prime} \cap W_{j} \neq \emptyset$. 
(c) There are distinct $W_{i_{1}}, W_{i_{2}}, W_{i_{3}}$ with $\left|W_{i_{1}}\right|,\left|W_{i_{2}}\right|,\left|W_{i_{3}}\right|<R$ and for every pair $j, j^{\prime} \in\{1,2,3\}$ such that $j \neq j^{\prime}$ a path of length at most $t$ from some $a \in W_{i_{j}}$ to some $b \in W_{i_{j^{\prime}}}$.

From the proofs of Lemmas 3.2, 3.3 and 3.5, it follows that the proportion of $\mathcal{C} \in \mathbf{C}\left(W_{1}, \ldots, W_{n}\right)$ satisfying any of (a), (b) or (c) approaches 0 as $n \rightarrow \infty$, and in each case the convergence is bounded by a function depending only on $R, k, r_{1}, \ldots, r_{k}$ and $s_{1}, \ldots, s_{k}$. Hence $\mathbb{E}\left(Z^{\prime \prime}\right)=o(1)$ as $n \rightarrow \infty$, where the bound depends only on $R, k, r_{1}, \ldots, r_{k}$ and $s_{1}, \ldots, s_{k}$.

Lemma 3.8 Suppose that $R \geq 3$. For every integer $p$, the proportion of $\mathcal{G} \in \mathbf{G}_{n, R}$ such that every connected component of $\mathcal{G}$ has at least $p$ vertices approaches 1 as $n \rightarrow \infty$.

Proof: Let $p$ be any positive integer. By Lemmas 3.3, 3.5, 3.6 and Theorem 2.1. almost all $\mathcal{G} \in \mathbf{G}_{R}$ have the following properties:

- There is no subgraph with $p$ vertices and more than $p$ edges.

- There do not exist distinct vertices $v_{i}, i=1,2,3$, all three with degree $R-1$ such that for all distinct $i, j \in\{1,2,3\}$ there is a path of length at most $p$ from $v_{i}$ to $v_{j}$.

- There do not exist distinct vertices $v$ and $w$ both of degree $R-2$ and a path of length at most $p$ from $v$ to $w$.

- No vertex has degree less than $R-2$.

Therefore it suffices to show that if $\mathcal{G} \in \mathbf{G}_{R}$ has a connected component with exactly $p$ vertices, then one of the above properties fail for $\mathcal{G}$. Recall the assumption that $R \geq 3$ and suppose that $\mathcal{H} \subseteq \mathcal{G}$ is a connected component with exactly $p$ vertices. If $p=1$ then the unique vertex in $\mathcal{H}$ has degree $0<R-2$, so $\mathcal{G}$ does not have the last property above. If $p=2$ then the two vertices of $\mathcal{H}$ have degree $1 \leq R-2$ in $\mathcal{G}$, so the third or fourth property fails. If $p=3$ then the three vertices of $\mathcal{H}$ have degree at most $2 \leq R-1$ in $\mathcal{G}$, so $\mathcal{G}$ does not have the second property. Now suppose that $p \geq 4$. If the last three properties hold, then the number of edges in $\mathcal{H}$ is at least

$$
\frac{(R-2)+(R-1)+(p-2) R}{2}=\frac{p R-3}{2} \geq p \frac{3}{2}-\frac{3}{2}>p,
$$

where the last inequality holds for all $p \geq 4$ (by induction or differentiation). Hence $\mathcal{H}$ has more than $p$ edges, so $\mathcal{G}$ does not have the first property.

Note that Lemma 3.8 is false for $R=2$, because if $R=2$ then every 3-cycle (say) is a connected component and the proportion of $\mathcal{G} \in \mathbf{G}_{n, 2}$ with at least one 3-cycle converges to a positive number, by Lemma 3.7, as $n \rightarrow \infty$. In the case $R \geq 5$ Lemma 3.8 is a consequence of Corollary 2.4.

\section{First-order limit laws}

Recall that $\mathbf{G}_{n, R}$ denotes the set of undirected graphs with vertices $1, \ldots, n$ such that every vertex has degree at most $R$. For every $n$ and $\mathcal{G} \in \mathbf{G}_{n, R}$ we let $[\mathcal{G}]$ denote the equivalence class to which it belongs with respect to the isomorphism relation on $\mathbf{G}_{n, R}$. Note that for every first-order sentence $\varphi$ and every $\mathcal{G} \in \mathbf{G}_{n, R}$, we have $\mathcal{G} \models \varphi$ if and only if $\mathcal{H} \models \varphi$ for every $\mathcal{H} \in[\mathcal{G}]$. 
Theorem 4.1 Let $R$ be a non-negative integer.

(i) Suppose that $R \geq 0$. For every first order sentence $\varphi$ in the language of graphs, there is $c \in[0,1]$ such that

$$
\lim _{n \rightarrow \infty} \frac{\left|\left\{\mathcal{G} \in \mathbf{G}_{n, R}: \mathcal{G} \models \varphi\right\}\right|}{\left|\mathbf{G}_{n, R}\right|}=c .
$$

(ii) Suppose that $0 \leq R \leq 1$ or $R \geq 5$. For every first-order sentence $\varphi$ in the language of graphs, there is $c \in[0,1]$ such that

$$
\lim _{n \rightarrow \infty} \frac{\mid\left\{[\mathcal{G}]: \mathcal{G} \in \mathbf{G}_{n, R} \text { and } \mathcal{G} \models \varphi\right\} \mid}{\left|\left\{[\mathcal{G}]: \mathcal{G} \in \mathbf{G}_{n, R}\right\}\right|}=\lim _{n \rightarrow \infty} \frac{\left|\left\{\mathcal{G} \in \mathbf{G}_{n, R}: \mathcal{G} \models \varphi\right\}\right|}{\left|\mathbf{G}_{n, R}\right|}=c .
$$

In other words, for every $R \geq 0$, finite graphs with maximum degree $R$ satisfy a labelled limit law for first-order logic. If $0 \leq R \leq 1$ or $R \geq 5$ then we also have an unlabelled limit law. If $R=0$ or $R=1$ then we have a zero-one law in both the labelled an unlabelled case (as the proof below will show), that is, the number $c$ in the theorem is either 0 or 1 for every $\varphi$. If $R \geq 2$ then we do not have a zero-one law, because (for example) the non-existence of a vertex with degree $R-2$, which can be expressed with a sentence in first-order logic, holds with asymptotic probability $e^{-(R-1)}$, by Theorem 2.1. This paper does not settle the question of whether or not $\mathbf{G}_{n, R}$ has an unlabelled limit law for $R=2,3,4$.

\subsection{Proof of Theorem 4.1}

If $R=0$ then $\left|\mathbf{G}_{n, R}\right|=1$ for every $n$, so the theorem is trivial in this case. If $R=1$ then there are only two isomorphism types of connected components, singletons and two vertices connected to each other, so by Example 7.15 in [5] the theorem follows, both in the labelled and unlabelled case. (In this simple case one can of course also argue directly, without reference to [5] which has much wider applicability.)

For the rest of the proof we assume that $R \geq 2$. We will prove that for every first-order sentence $\varphi$, the proportion $\left|\left\{\mathcal{G} \in \mathbf{G}_{n, R}: \mathcal{G}=\varphi\right\}\right| /\left|\mathbf{G}_{n, R}\right|$ converges as $n \rightarrow \infty$; so we get part (i) of the theorem for all $R \geq 2$. By Corollary 2.4 and Theorem 2.3 we then get part (ii) for $R \geq 5$, because in Theorem 2.3 we can, for any sentence $\varphi$, let $\mathbf{H}_{n}$ be the set of $\mathcal{G} \in \mathbf{G}_{n, R}$ such that $\mathcal{G}=\varphi$.

Every first-order sentence has a quantifier rank (also called quantifier depth) (see [6, 11, 16] for example) which is a non-negative integer. Therefore it suffices to show that for every $k>0$ and every first-order sentence $\varphi$ with quantifier rank at most $k$, the quotient $\left|\left\{\mathcal{G} \in \mathbf{G}_{n, R}: \mathcal{G} \models \varphi\right\}\right| /\left|\mathbf{G}_{n, R}\right|$ converges as $n \rightarrow \infty$. So we fix an arbitrary integer $k \geq 3$.

Definition 4.2 Let $m=5^{k+1}$. For all $q, r_{3}, \ldots, r_{m}, s_{1}, \ldots, s_{m} \in\{0,1, \ldots, k\}$, define

$$
\mathbf{X}_{n}\left(q, r_{3}, \ldots, r_{m}, s_{1}, \ldots, s_{m}\right)
$$

to be the set of all $\mathcal{G} \in \mathbf{G}_{n, R}$ such that the following holds:

1. There are no vertices with degree less that $R-2$,

2. If $q<k$ then there are exactly $q$ vertices with degree $R-2$ and if $q=k$ then there are at least $k$ vertices with degree $R-2$. 
3. There are at least $m$ vertices with degree $i$ for $i=R-1, R$.

4. For $p=3, \ldots, m$, if $r_{p}<k$ then there are exactly $r_{p} p$-cycles and if $r_{p}=k$ then there are at least $k p$-cycles.

5. For $p=1, \ldots, m$, if $s_{p}<k$ then there are exactly $s_{p} p$-paths with both endpoints of degree $R-1$ and if $s_{p}=k$ then there are at least $k p$-paths with both endpoints of degree $R-1$.

6. The distance is at least $5^{k+2}$ between

(a) any vertex with degree $R-2$ and any (other) vertex of degree at most $R-1$,

(b) any vertex with degree at most $R-1$ and any cycle of length at most $m$,

(c) any two different cycles of length at most $m$, and

(d) any two different paths of length at most $m$ such that both endpoints of both paths have degree $R-1$.

7. If $R \geq 3$ then every connected component has at least $m$ vertices.

Let

$$
P_{k}(x, \mu)=\left\{\begin{array}{l}
\frac{\mu^{x} e^{-\mu}}{x !} \quad \text { if } x<k, \\
1-\sum_{i=0}^{k-1} \frac{\mu^{i} e^{-\mu}}{i !} \quad \text { if } x \geq k,
\end{array}\right.
$$

and let $\lambda_{p}=\frac{(R-1)^{p}}{2 p}$ and $\mu_{p}=\frac{(R-1)^{p+1}}{2}$. By Theorems 2.1 and 3.1 and Lemma 3.7 .

$$
\begin{aligned}
\lim _{n \rightarrow \infty} \frac{\left|\mathbf{X}_{n}\left(q, r_{3}, \ldots, r_{m}, s_{1}, \ldots, s_{m}\right)\right|}{\left|\mathbf{G}_{n, R}\right|} \\
\quad=P_{k}(q, R-1)\left(\prod_{p=3}^{m} P_{k}\left(r_{p}, \lambda_{p}\right)\right)\left(\prod_{p=1}^{m} P_{k}\left(s_{p}, \mu_{p}\right)\right) .
\end{aligned}
$$

Hence it is enough to prove that for all $q, r_{3}, \ldots, r_{m}, s_{1}, \ldots, s_{m} \in\{0,1, \ldots, k\}$, if

$$
\mathcal{G}, \mathcal{H} \in \mathbf{X}_{n}\left(q, r_{3}, \ldots, r_{m}, s_{1}, \ldots, s_{m}\right),
$$

then $\mathcal{G}$ and $\mathcal{H}$ satisfies exactly the same sentences of quantifier rank at most $k$. To show that $\mathcal{G}$ and $\mathcal{H}$ satisfies exactly the same sentences of quantifier rank at most $k$ it suffices to prove that Duplicator has a winning strategy for the Ehrenfeucht-Fraïssé game in $k$ steps on $\mathcal{G}$ and $\mathcal{H}$ (e.g. [6], Theorem 2.2.8, or similar results in [11, 16]).

So we fix $q, r_{3}, \ldots, r_{m}, s_{1}, \ldots, s_{m} \in\{0,1, \ldots, k\}$ and let

$$
\mathcal{G}, \mathcal{H} \in \mathbf{X}_{n}\left(q, r_{3}, \ldots, r_{m}, s_{1}, \ldots, s_{m}\right) .
$$

For any vertex $v$ of $\mathcal{G}$ and $l \in \mathbb{N}_{0}$ we let $B_{\mathcal{G}}(v, l)$ be the set of vertices $w$ such that $\operatorname{dist}_{\mathcal{G}}(v, w) \leq l$; and similarly for $\mathcal{H}$. Let a Poisson object of $\mathcal{G}$ or $\mathcal{H}$ denote any one of

- a vertex with degree $R-2$, or 
- a $p$-cycle where $p \leq m$, or

- a $p$-path with both endpoints of degree $R-1$ where $p \leq m$.

Observation 4.3 It follows from the definition of $\mathbf{X}_{n}\left(q, r_{3}, \ldots, r_{m}, s_{1}, \ldots, s_{m}\right)$ that if $v \in\{1, \ldots, n\}$, $B=B_{\mathcal{G}}(v, l)$, or $B=B_{\mathcal{H}}(v, l)$, and $l \leq 5^{k}$, then $B$ contains at most one Poisson object.

To prove that Duplicator has a winning strategy for the Ehrenfeucht-Fraïssé game in $k$ steps on $\mathcal{G}$ and $\mathcal{H}$ it suffices to prove the following result.

Lemma 4.4 Suppose that $i<k, v_{1}, \ldots, v_{i}, w_{1}, \ldots, w_{i} \in\{1, \ldots, n\}, v_{i+1} \in\{1, \ldots, n\}$ (or $w_{i+1} \in$ $\{1, \ldots, n\})$ and that

$$
f_{i}: \mathcal{G}\left[\bigcup_{j=1}^{i} B_{\mathcal{G}}\left(v_{i}, 5^{k-i}\right)\right] \rightarrow \mathcal{H}\left[\bigcup_{j=1}^{i} B_{\mathcal{H}}\left(w_{i}, 5^{k-i}\right)\right]
$$

is an isomorphism such that $f_{i}\left(v_{j}\right)=w_{j}$ for $j=1, \ldots, i$. Then there is $w_{i+1} \in\{1, \ldots, n\}$ (or $v_{i+1} \in$ $\{1, \ldots, n\}$ ) such that the same statement holds with ' $i+1$ ' in place of ' $i$ '.

Proof: Suppose that $i<k, v_{1}, \ldots, v_{i}, w_{1}, \ldots, w_{i} \in\{1, \ldots, n\}$ and that

$$
f_{i}: \mathcal{G}\left[\bigcup_{j=1}^{i} B_{\mathcal{G}}\left(v_{i}, 5^{k-i}\right)\right] \rightarrow \mathcal{H}\left[\bigcup_{j=1}^{i} B_{\mathcal{H}}\left(w_{i}, 5^{k-i}\right)\right]
$$

is an isomorphism such that $f_{i}\left(v_{j}\right)=w_{j}$ for $j=1, \ldots, i$. By symmetry it is enough to consider the case when Spoiler chooses $v_{i+1}$ in $\mathcal{G}$.

If $B_{\mathcal{G}}\left(v_{i+1}, 5^{k-i-1}\right) \subseteq \bigcup_{j=1}^{i} B_{\mathcal{G}}\left(v_{j}, 5^{k-i}-1\right)$ then let $w_{i+1}=f_{i}\left(v_{i+1}\right)$ and let $f_{i+1}$ be the restriction of $f_{i}$ to $\bigcup_{j=1}^{i+1} B_{\mathcal{G}}\left(v_{j}, 5^{k-i-1}\right)$.

Now suppose that $B_{\mathcal{G}}\left(v_{i+1}, 5^{k-i-1}\right)$ is not included in $\bigcup_{j=1}^{i} B_{\mathcal{G}}\left(v_{j}, 5^{k-i}-1\right)$. Then there is $u \in$ $B_{\mathcal{G}}\left(v_{i+1}, 5^{k-i-1}\right)$ such that the distance (in $\mathcal{G}$ ) from $u$ to $v_{j}$ is at least $5^{k-i}$ for all $j=1, \ldots, i$. It follows that for every $a \in B_{\mathcal{G}}\left(v_{i+1}, 5^{k-i-1}\right)$ and every $j=1, \ldots, i$, the distance from $a$ to $v_{j}$ is at least

$$
5^{k-i}-\operatorname{dist}_{\mathcal{G}}(a, u) \geq 5^{k-i}-2 \cdot 5^{k-i-1}=3 \cdot 5^{k-i-1} .
$$

Consequently, for every $a \in B_{\mathcal{G}}\left(v_{i+1}, 5^{k-i-1}\right)$ and every $b \in \bigcup_{j=1}^{i} B_{\mathcal{G}}\left(v_{j}, 5^{k-i-1}\right)$,

$$
\operatorname{dist}_{\mathcal{G}}(a, b) \geq 3 \cdot 5^{k-i-1}-5^{k-i-1}=2 \cdot 5^{k-i-1} \geq 2
$$

because $i<k$. Since $\mathcal{G}, \mathcal{H} \in \mathbf{X}_{n}\left(q, r_{3}, \ldots, r_{m}, s_{1}, \ldots, s_{m}\right)$ and $f_{i}$ is an isomorphism it follows from Observation 4.3 for large enough $n$, that there are $w_{i+1}$ and an isomorphism

$$
f_{i+1}^{\prime}: \mathcal{G}\left[B_{\mathcal{G}}\left(v_{i+1}, 5^{k-i-1}\right)\right] \rightarrow \mathcal{H}\left[B_{\mathcal{H}}\left(w_{i+1}, 5^{k-i-1}\right)\right]
$$

such that $f_{i+1}^{\prime}\left(v_{i+1}\right)=w_{i+1}$ and $\operatorname{dist}_{\mathcal{H}}\left(w_{i+1}, u\right) \geq 2$ for every $u \in \bigcup_{j=1}^{i} B_{\mathcal{H}}\left(w_{i}, 5^{k-i}\right)$. Then the desired isomorphism $f_{i+1}$ is obtained by letting $f_{i+1}(v)=f_{i}(v)$ if

$v \in \bigcup_{j=1}^{i} B_{\mathcal{G}}\left(v_{i}, 5^{k-i}\right)$ and $f_{i+1}(v)=f_{i+1}^{\prime}(v)$ if $v \in B_{\mathcal{G}}\left(v_{i+1}, 5^{k-i-1}\right)$. 


\section{Acknowledgements}

I thank Svante Janson and Nicholas Wormald for helpful comments and discussions. In particular, Janson suggested the proof of Theorem 2.1 which is presented here. (My proof first obtained some rough estimates by counting arguments and then applied a known asymptotic formula for graphs with given degree sequence, which, however, involved rather long and tedious calculations.) Also, thanks to the anonymous referee for careful reading and helping me to remove various mistakes.

\section{References}

[1] E. A. Bender, Asymptotic methods in enumeration, SIAM Review, Vol. 16 (1974) 485-515.

[2] E. A. Bender, E. R. Canfield, The asymptotic number of labelled graphs with given degree sequences, Journal of Combinatorial Theory, Ser. A, Vol. 24 (1978) 296-307.

[3] B. Bollobás, A probabilistic proof of an asymptotic formula for the number of labelled regular graphs, European Journal of Combinatorics, Vol. 1 (1980) 311-316.

[4] B. Bollobás, Random Graphs, Second Edition, Cambridge University Press, 2001.

[5] K. J. Compton, A logical approach to asymptotic combinatorics I. First order properties, Advances in Mathematics, Vol. 65 (1987) 65-96.

[6] H-D Ebbinghaus, J. Flum, Finite Model Theory, Second Edition, Springer (1999).

[7] S. Haber, M. Krivelevich, The logic of random regular graphs, Journal of Combinatorics, Vol. 1 (2010) 389-440.

[8] C. Hundack, H. J. Prömel, A. Steger, Extremal graph problems for graphs with a color-critical vertex, Combinatorics, Probability and Computing, Vol. 2 (1993) 465-477.

[9] J. W. Kennedy, L. V. Quintas, Probability models for random $f$-graphs, in Combinatorial Mathematics: Proceedings of the Third International Conference, Annals of the New York Academy of Sciences, Vol. 555 (1989) 248-261.

[10] V. Koponen, A limit law of almost $l$-partite graphs, submitted. Available via: http://arxiv.org/abs/1204.2454

[11] L. Libkin, Elements of Finite Model Theory, Springer (2004).

[12] J. F. Lynch, Convergence law for random graphs with specified degree sequence, ACM Transactions on Computational Logic, Vol. 6 (2005) 727-748.

[13] B. D. McKay, N. C. Wormald, Automorphisms of random graphs with specified vertices, Combinatorica, Vol. 4 (1984) 325-338.

[14] A. Rucinski, N. C. Wormald, Random graph processes with degree restrictions, Combinatorics, Probability and Computing, Vol. 1 (1992) 169-180. 
[15] A. Rucinski, N. C. Wormald, Random graph processes with maximum degree 2, The Annals of Probability, Vol. 7 (1997) 183-199.

[16] J. Spencer, The strange logic of random graphs, Springer (2001).

[17] N. C. Wormald, The asymptotic connectivity of labelled regular graphs, Journal of Combinatorial Theory, Series B, Vol. 31 (1981) 156-167.

[18] N. C. Wormald, The asymptotic distribution of short cycles in random regular graphs, Journal of Combinatorial Theory, Series B, Vol. 31 (1981) 168-182.

[19] N. C. Wormald, Models of random regular graphs, in J. Lamb and D. Preece (editors), Surveys in Combinatorics, 1999, Vol. 276 of London Mathematical Society Lecture Notes Series, 239-298, Cambridge University Press, 1999. 